\title{
An Innovative Framework for Real Time Monitoring of Pollutant Point Sources in River Networks
}

\section{BaratiMoghaddam}

Tarbiat Modares University https://orcid.org/0000-0001-8346-1707

Mehdi Mazaheri ( $\nabla$ m.mazaheri@modares.ac.ir)

Tarbiat Modares University https://orcid.org/0000-0001-8670-1710

\section{J. M. V. Samani}

Tarbiat Modares University

Fulvio Boano

Politecnico di Torino

\section{Research Article}

Keywords: Geostatistical approach, Inverse problem, Multiple pollutant point sources, Source identification, Unsteady flow, River network

Posted Date: June 18th, 2021

DOl: https://doi.org/10.21203/rs.3.rs-597673/v1

License: (c) (1) This work is licensed under a Creative Commons Attribution 4.0 International License. Read Full License 
1 An Innovative Framework for Real Time Monitoring of Pollutant

2

3

4

5

6

7

8

9

10

11

12

13

14

15

16

17

18

19

20

21

\section{Point Sources in River Networks}

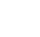

M. BaratiMoghaddam ${ }^{1,2}$, M. Mazaheri ${ }^{1 *}$, J. M. V. Samani ${ }^{1}$ and F. Boano $^{2}$

${ }^{1}$ Department of Water Engineering and Management, Tarbiat Modares University, Tehran, Iran.

${ }^{2}$ Department of Environment, Land and Infrastructure Engineering (DIATI), Politecnico di Torino, (1) Turin, Italy.

(1)

(1)

* Corresponding author: m.mazaheri@ modares.ac.ir

9

10




\section{Point Sources in River Networks}

\section{Abstract:}

The simultaneous identification of location and source release history in complex river networks is a very complicated ill-posed problem, particularly in a case of multiple unknown pollutant sources with time-varying release pattern. This study presents an innovative method for simultaneous identification of the number, locations and release histories of multiple pollutant point sources in a river network using minimum observation data. Considering two different type of monitoring stations with an adaptive arrangement as well as real-time data collection at those stations and using a reliable numerical flow and transport model, at first the number and suspected reach of presence of pollutant sources are determined. Then the source location and its intensity function is calculated by solving inverse source problem using a geostatistical approach. A case study with three different scenarios in terms of the number, release time and location of pollutant sources are discussed, concerning a river network with unsteady and non-uniform flow. Results showed the capability of the proposed method in identifying of sought source characteristics even in complicated cases with simultaneous activity of multiple pollutant sources.

40 Source identification, Unsteady flow, River network. 


\section{1. Introduction}

Water resources are essential to life on the Earth planet, but these limited and valuable resources are increasingly under threat. Rivers in particular due to proximity to big cities and extensive usage in industrial and agricultural activities, are extremely exposed to accidental or intentional spills. Regarding to this issue, in recent years, a great attention has been drawn to simulate fate and transport of contaminants in rivers as well as to identify pollutant sources characteristics. Recovering release history of pollutant sources is essential in planning effective remediation strategies. Moreover, determining the number and location of pollutant sources is of great importance in order to identify responsible parties for observed pollution cloud in downstream and divide remediation measure expenses among those parties (Skaggs and Kabala, 1994, Liu and Ball, 1999, Atmadja and Bagtzoglou, 2001, Michalak, 2002).

Given known concentration data at limited downstream observation points, the pollutant source identification problem is categorized as an inverse problem. Like most of the inverse problems, the inverse source problem does not fulfill the well-posedness criteria of Hadamard (1923). Based on Hadamard's definition, a problem is well-posed if its solution is existent, unique and stable. A problem which lacks any of these features called an ill-posed problem. However, since the observed pollution cloud at the downstream point, must be originated from somewhere at the upstream, pollutant source identification problem always has a solution and nonexistence would not raise an issue. Hence, there are two main challenges in solving an inverse source problem, namely nonuniqueness and instability of the solution. The nonuniqueness means that different combinations of intensity functions of the pollution sources at the upstream can create a single concentration-time curve at a given observation point downstream. Since time discretization of governing equation to pollution transport results in a system of equations which has fewer equations (observations) than unknowns (source values), multiple combinations for source characteristics might be detected which are consistent with 
observed concentration data. To address the nonuniqueness issue researchers often assume that some prior information about the unknown source is available (e.g. possible location, activity duration or known intensity function, as well as, consideration a particular form of the source term function). The instability issue implies to large errors in the solution following small errors in measured data. It is mainly a consequence of irreversibility of dispersion phenomena, which gradually smooth the pollution plume and decrease the amount of obtainable information from observational data (Skaggs and Kabala, 1998). Hence, considering uncertainties in observed data regarding to measurement errors and sparsity of data increases the reliability of the identification results.

In the last 30 years, various methods have been proposed to solve pollutant source identification problem in surface and groundwater which can be broadly categorized into three classes: optimization-based approaches, stochastic-based approaches and mathematics-based approaches. A review of those research can be found in (Atmadja and Bagtzoglou, 2001, Michalak and Kitanidis, 2004b, Morrison, 2000b, Morrison, 2000a, Neupauer et al., 2000). Among these methods, stochastic-based methods are becoming a trend in solving inverse source problem in recent years. The most significant feature of stochastic-based approaches is to treat unknown pollution source parameters as random variables and use of probability distribution functions to predict those parameters. This feature provides the possibility of estimation of the source characteristics in greater number of instants than available observation data as well as consideration of uncertainty due to error in those data (Woodbury et al. 1998).

One of the stochastic-based methods which is extensively used in solving of inverse source problem in groundwater is Geostatistical (GS) method. The main assumption of GS method is that the unknown source function is random with a known correlation structure but unknown correlation structural parameters. The optimal values of these structural parameters are obtained using the geostatistical inversion theory presented by Kitanidis (1996) and the source 
94 function recovered by minimizing a likelihood function while retaining the assumed correlation structure. More details can be found in Kitanidis $(1995,1996)$ and Snodgrass and Kitanidis (1997). The GS approach has been widely tested and improved in groundwater source identification through hypothetical cases (Snodgrass and Kitanidis, 1997, Michalak and Kitanidis, 2003, Michalak and Kitanidis, 2004b, Butera et al., 2013) and using field data (Michalak and Kitanidis, 2002, Michalak and Kitanidis, 2004a, Gzyl et al., 2014). This approach also had been applied only once in pollutant source identification in single-branched rivers considering the effects of transient storage zone as well as linear decay processes (Boano et al., 2005). Snodgrass and Kitanidis (1997) applied the GS approach for estimating the release history of a conservative solute in a 1D homogeneous aquifer. Instead of using the usual iterative techniques to obtain the best estimation of parameters, they combined GS techniques with Bayesian theory, which provides the possibility to quantify the estimation error. Michalak and Kitanidis (2002) applied the proposed approach by Snodgrass and Kitanidis (1997) for the reconstruction of the contaminant release history for a 3D plume at Gloucester landfill site in Ontario, Canada. Michalak and Kitanidis (2004b) combined the adjoint model with geostatistical techniques in order to reduce the computational cost as well as providing the possibility to use the approach in heterogeneous fields. In addition, using an adjoint model provides the feasibility of application of the existing groundwater flow and transport commercial codes in the framework of the proposed inverse method. Butera et al. (2013) based on GS proposed a framework for simultaneous identification of location and release history of a single pollutant source in 2D confined aquifers with strongly non-uniform flow field. Gzyl et al. (2014) presented a multi-step method based on performing an integral pumping test and GS approach to identify location and release history of a pollutant source in groundwater. The results of applying this method to a complicated contamination case at the adjacent reach to an abandoned chemical plant in southern Poland, indicated that it is able to successfully detect 
119 suspected areas. However, the proposed methods by Butera et al. (2013) and Gzyl et al. (2014)

120 need the prior knowledge of the approximate location of pollutant source at the beginning of a

121 simulation, which is a challenge in practical applications, especially in complicated cases that

122 such information may not be available.

123 Compared to numerous studies on pollutant source identification of in groundwater, only

124 relatively few studies on solving an inverse source problem in surface waters can be found in

125 literature (El Badia and Hamdi, 2007, Hamdi, 2009, Hamdi, 2016, Andrle and El Badia, 2012,

126 Cheng and Jia, 2010, Mazaheri et al., 2015, Yang et al., 2016, Wang et al., 2018). While, the

127 pollutant transport in rivers tends to be more advection-dominated than groundwater and,

128 subsequently pollutant substance transported faster and further, which may lead to partial

129 capturing of the pollution plume at the observation points. Thus, fast and accurate identification

130 of illegal spills is more important in these environments to provide scientific support for

131 planning mitigation and adaptation strategies. Furthermore, the research of pollutant source

132 identification problem in surface waters was mainly confined to single-branch rivers and rarely

133 involved river networks. This is mainly due to hydrodynamical complexity of such systems

134 which along with inherent ill- posedness of corresponding source identification problem, form

135 a problem that is very difficult to solve. However, regarding that tributaries in a river network

136 usually are less monitored, those areas might be considered as potential places for illegal

137 discharge of pollutants. Therefore, to prevent further damage, it is necessary to pay more

138 attention to identifying pollutant sources characteristics in such environments.

139 Focusing on pollutant source identification in river networks, Telci and Aral (2011) by

140 using an adaptive sequential feature selection algorithm (Jiang, 2008), determined the location

141 of a single instantaneous source among several candidate locations. However, their proposed

142 method requires a significant amount of simulation time for training monitoring stations with

143 a large number of spill scenarios. Ghane et al. (2016) applied the backward probability method 
144 to identify the source location and the released time of a single spill in a river system. Lee et

145 al. (2018) dealt with the problem of identifying the location of a single instantaneous source

146 via analyzing changes in concentration levels that observed by a sensor network in a river

147 system. By constructing random forest models, they determined the possibility that each

148 candidate location be the correct one as a number between zero and one. However, all of

149 mentioned studies considered a single pollutant source with a simple form of release (i.e. the

150 spill), while in many practical application, there are more than one active source and the release

151 functions varies with time.

152 Apart from the issue of insufficient studies on pollution source identification in rivers, most

153 of previous studies considered the location of the pollutant source to be known priori. This

154 assumption is not compatible with real-world condition, since in most cases the location of the

155 pollution source is also unknown as its intensity function. Introducing the source location as an

156 unknown, will have a significant effect on source identification process due to interaction

157 between a release at a variable source location and observational data. In other words, different

158 potential source location sets may result in significantly different solutions. Moreover, the

159 simultaneous identification of location and source release history is a very complicated ill-

160 posed problem, particularly in a case of multiple unknown pollution sources with time-varying

161 release pattern. The main motivations behind this study is to provide an innovative method for

162 simultaneous identification of the number, locations and release histories of multiple point

163 sources in a river network using minimum observational data and considering near real world

164 conditions namely unsteady and non-uniform flow as well as reactive pollutants. The proposed

165 method includes two main steps that are given below:

166 Step1: determining the number and suspected reaches to presence of sources by placement of

167 observation points in a specific manner and management of data collection at those stations. 
Step 2: identification of exact location and intensity function of the source by solving the inverse source problem using a geostatistical approach.

170 The method is effective and easy to apply in complex river networks as well as single-branch

171 ones. Moreover, it provides the possibility of simulators identification of all active pollutant

172 sources. Hence the required computational time is significantly lower than common iterative

173 methods such as simulation-optimization approach.

\section{2. Material and Methods}

\subsection{Governing Equations and Statement of the problem}

176 The main governing equation of solute transport in surface waters is advection-dispersion

177 equation (ADE) (Taylor, 1954), which is a parabolic partial differential equation derived from

178 a combination of continuity equation and Fick's first law. The one-dimensional ADE equation 179 is as follows (Fischer et al., 1979):

$$
\frac{\partial(A C)}{\partial t}+\frac{\partial(C Q)}{\partial x}-\frac{\partial}{\partial x}\left(A D \frac{\partial C}{\partial x}\right)+A \lambda C-\sum_{i=1}^{m} f_{i}(t) \delta\left(x-x_{i}\right)=0
$$

where, $A$ is the flow area, $C$ is the solute concentration, $Q$ is the volumetric flow rate, $D$ is the dispersion coefficient, $\lambda$ is the first-order decay coefficient, $m$ is the number of pollution

184 respectively. It also should be mentioned that, hydrodynamic parameters (i.e., $A, Q, D$ ) in

185 Equation (1) are obtained from the hydrodynamics model which is based on well-established

186 Saint-Venant equations (Wu, 2007):

$$
\begin{aligned}
& \frac{\partial A}{\partial t}+\frac{\partial Q}{\partial x}=0 \\
& \frac{\partial Q}{\partial t}+\frac{\partial}{\partial x}\left(\frac{Q^{2}}{A}\right)+g A \frac{\partial z_{s}}{\partial x}+g A S_{f}=0
\end{aligned}
$$

187 in which $\mathrm{z}_{\mathrm{s}}$ and $S_{f}$ are water level and energy slope, respectively. 
The general expression of the considered problem is that there are multiple pollutant point

189 sources $S_{1}, S_{2}, \mathrm{~K}, S_{m}$ in a river network, which the number, locations $\left(x_{1}, x_{2}, \ldots \ldots . ., x_{m}\right)$ and 190 intensity functions $\left(f_{1}(t), f_{2}(t), \ldots \ldots ., f_{m}(t)\right)$ of those sources are unknown. The main 191 objectives are to present a methodology for simultaneous identification of these sources 192 characteristics (i.e. their number, locations, and intensity functions), and obtaining a unique response for the considered inverse source problem with a minimum measured concentration data at observation points. The proposed method consists of two main steps. The method starts with the determination of a spatial range in which the source of pollution is likely to be present. Then the location and approximate release history of pollution sources are recovered by means of a geostatistical approach, that considered simultaneously all the possible candidates. The method is effective and easy to apply in complex river networks as well as single-branch ones. Moreover, since in each simulation all active pollutant sources are identified, the required computational time is significantly lower than common iterative methods such as simulationoptimization approach. More details are given in following sections.

\subsection{Step1: Determination of the Number and Approximate Location of Pollution} Sources

In order to determine the approximate location of pollutant sources, some observation points are considered with a specific arrangement and data collection at those observation points are managed based on specified condition of each problem. In order to provide the concentration data and proceed with the identification process, two types of observation points are defined, main $\left(M_{1}, M_{2}\right.$ $\left.M_{n}\right)$ and secondary stations stations collect concentration-time data continuously, but the secondary ones collect data occasionally and on-demand. The placement of main and secondary stations is based on some

211 priori information including desired activity time for retrieval and accuracy of spatial range for 
212 pollution source localization. The main stations are placed in a way that the travel time between

213 two successive main stations always is less than or equal to the expected activity time for the

214 sources. The travel time between successive main stations for each branch of the river network

215 and is calculated using following equation (Chapra, 2008):

$$
\bar{T}=\frac{\sum_{i=1}^{n-1}\left(C_{i} t_{i}+C_{i+1} t_{i+1}\right)\left(t_{i+1}-t_{i}\right)}{\sum_{i=1}^{n-1}\left(C_{i}+C_{i+1}\right)\left(t_{i+1}-t_{i}\right)}
$$

216 in which $\bar{T}$ is travel time, $C_{i}$ is the concentration at temporal instant $t_{i}$. The secondary stations

217 are arranged in a way that the distance between two successive stations be equal to the accuracy

218 which expected for approximate location of the sources. This configuration of monitoring 219 station makes it possible to identify all active pollutant sources with minimum measurement 220 data and avoid additional data collection as well as related costs.

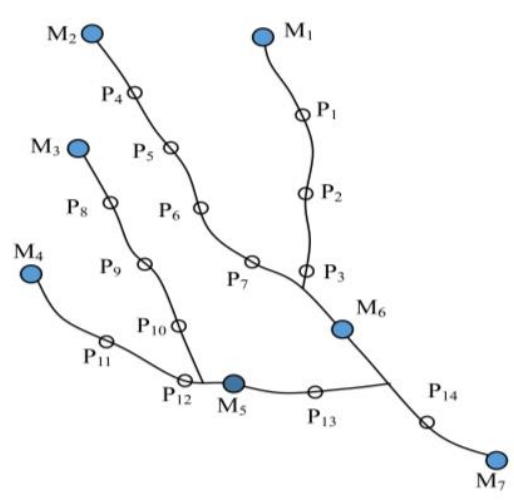

Figure 1- A hypothetical river network and arrangement of main and secondary stations

223 in the main stations, so that any difference between these two sets of data is a sign of existence 224 of a pollutant source at the upstream of that particular station. The simulated data are taken 225 from an integrated flow and transport model, which solves equation (1) - (3) in a river network for a case of no active pollutant source. It is a real-time simulation model which continuously 
executed and its outputs namely concentration-time data $C(x, t)$ are used in solving the inverse source problem by proposed algorithm.

Once a main station detects a difference between observed and simulated concentration data, depending on the type of communication topology of monitoring system a command will be send from a control center or directly from that main station to all secondary stations which have been located between that main station and the first main station at upstream of it, to collect a concentration data at the instant of difference detection. The first secondary station

234 from upstream which shows a difference between observed and simulated data, guide us to the approximate location of the source. In other words, the pollution source must be located in the reach between that secondary station and the first secondary station at its upstream (Figure 2). After determining the approximate location of the pollution source, following actions should be done:

1. The secondary station which detected the difference as well as the secondary station source.

247 It also should be noted that the continuous data collecting at secondary stations, which frame 248 the source location, will be stopped after the full passage of pollution cloud from secondary 249 station that located at the downstream bound of suspected reach. The identification process of 250 approximate location of a case with multiple pollutant sources, are quite similar to what was described for the case with one active source (Figure 3). 

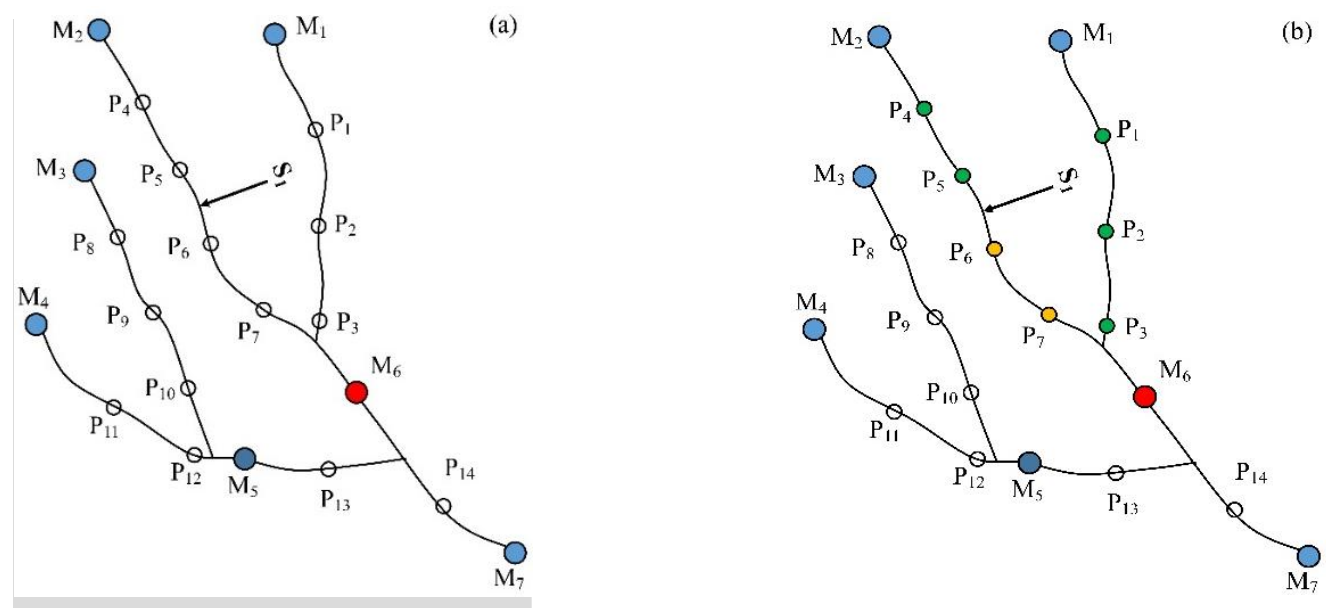

Figure 2- detection of approximate location in the case of one active source

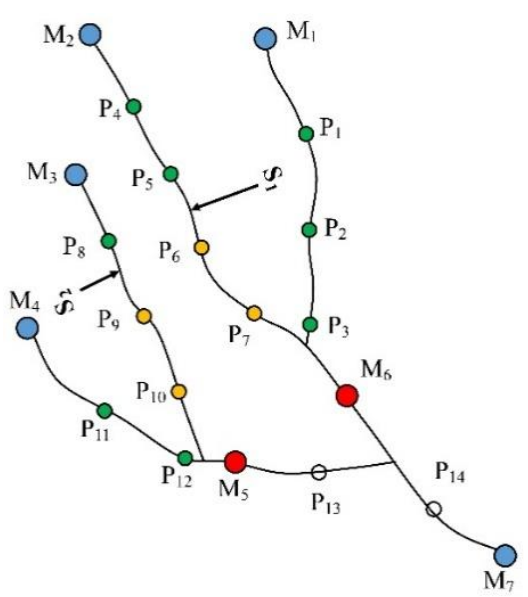

Figure 3- A case with two active sources

\subsection{Step 2: Recovering the Characteristics of Pollutant Sources by}

\section{Means of a Geostatistical Method}

256 After determining the number and suspected reaches to presence of pollution sources, the exact

257 location and approximate intensity function of pollution sources should be determined. Hence,

258 at first the mentioned reaches are divided to some sub-reaches and the potential location of

259 pollutant sources are considered at the center of those sub-reaches. Then, by solving an inverse source problem, the true location of the pollutant sources (i.e., where the pollutant injection has most likely originated) is determined as a location that the highest contaminant release history

262 is obtained. In order to solve the inverse source problem a Geostatistical method (GS) has been

263 used in this study. Regarding the linearity of equation (1) the solution of these equation subject 
264 to initial and boundary conditions (i.e., $\left.C(x, 0)=C_{0}(x), C(0, t)=C_{i n}(t), C(L, t)=0\right)$ is 265 (Skaggs and Kabala, 1994):

$$
C(\mathbf{x}, t)=\int_{0}^{t} f(\tau) K(\mathbf{x}, t-\tau) d \tau
$$

266 where $K(\mathbf{x}, t-\tau)$ is the transfer function (TF), that describes the effect in time at a certain

267 location $\mathrm{x}$ by a unitary impulse source which is released at $\mathrm{x}_{0}$ and time $\tau$. If $M$ observational

268 data be available and the time domain is discretized in $N$ instants, a general expression of the

269 relation between the observations and the source can be written as follows:

$$
\mathbf{z}=\mathbf{h}(\mathbf{f})+\mathbf{v}
$$

where $\mathbf{z}$ is a $[M \times 1]$ random vector of observations, $\mathbf{f}$ is a $[N \times 1]$ random vector of discretized

271 release history, $\mathbf{h}$ is the model function and $\mathbf{v}$ is a $[M \times 1]$ random vector that represents the

measurement errors. The error vector $\mathbf{v}$ is Gaussian with a zero mean and a covariance matrix as $\mathrm{R}=\sigma_{R}^{2} \mathrm{I}$ in which $\mathbf{I}$ is the $[M \times M]$ identity matrix. It also should be noted that $N ? M$, which means that there are more unknowns than measurements. By comparing equation (6) and (5) it can be concluded that the function $\mathbf{h}(\mathbf{f})$ is linear and therefore equation (6) can be rewritten as follows:

$$
\mathbf{Z}=\mathbf{H} \mathbf{f}+\mathbf{v}
$$

277 where $\mathbf{H}$ is a $[M \times N]$ matrix known as transfer matrix and its generic element is:

$$
H_{i, j}=\Delta \tau \begin{cases}K\left(t_{i}-\tau_{j}\right) & t_{i}>\tau_{j} \\ 0 & t_{i}<\tau_{j}\end{cases}
$$

278 in which $\Delta \tau$ is time step between two successive discretization of intensity function, $t_{i}$ and $\tau_{j}$

279 are observation instants and release time, respectively.

280 The $H_{i, j}$ element of transfer matrix represents the effect of a release at $\tau_{j}$ on observation 281 data $z_{i}$ at which collected at $t_{i}$. As shown in equation (8), to construct the $\mathbf{H}$ matrix, it is 282 necessary to calculate TFs at different time instants. TFs describe the response of the system 
to a unit impulse injection. Therefore, to calculate them, the ADE equation (equation (1)) need

to be solved for a unitary release function at the source location and for different time instants.

In case of simple problems with steady flow, regular cross-sections and constant parameters,

TFs can be determined using analytical procedures. However, in many practical applications,

with unsteady flow, irregular cross-sections and variable parameters using analytical formulas in evaluation of TFs values, is only possible by considering a series of simplifying assumptions.

As a consequence, a rough approximation in the solution of inverse problem expected, that is not desirable. Due to the complex conditions that considered in this study, the transfer functions have been calculated using the finite volume numerical method. To calculate the value of $H_{i, j}$ terms, several runs of the numerical model were performed. In case of unsteady

$$
H_{i j}=C\left(x, t_{i}\right)=\int_{0}^{t_{i}} \delta\left(\tau_{j}\right) K\left(x, t_{i}-\tau_{j}\right) d \tau
$$

297 The equation (7) is a system of ill-posed equations that cannot be solved by conventional methods. In order to overcome this difficulty, it is assumed that $\mathbf{f}$ has a normal distribution with mean and covariance as follows:

$$
\begin{aligned}
& E[\mathbf{f}]=\mathbf{X} \beta \\
& E\left[(\mathbf{f}-\mathbf{X} \beta)(\mathbf{f}-\mathbf{X} \beta)^{T}\right]=\mathbf{Q}(\boldsymbol{\theta})
\end{aligned}
$$

300 where $\mathbf{X}$ is a $[N \times 1]$ unit vector, $\beta$ is the unknown mean, $\boldsymbol{\theta}$ is a vector of unknown structural

301 parameters of the covariance function, and $\mathbf{Q}$ is the covariance matrix of the release $f(\tau)$. In

302 this research, a Gaussian covariance matrix has been considered, whose formulation is as 303 follows: 
$\mathbf{Q}\left(\tau_{i}-\tau_{j} \mid \boldsymbol{\theta}\right)=\sigma^{2} \exp \left[-\frac{\left(\tau_{i}-\tau_{j}\right)^{2}}{I_{f}{ }^{2}}\right]$

304 where $\theta^{T}=\left[\sigma^{2}, I_{f}\right]$ are structural parameters.

305 The reconstruction of pollutant source intensity function in the geostatistical method consist 306 of two steps. In the first step, known as structural analysis, the structural parameters of the

307 covariance function $\theta$ are determined, and in the second step, the contaminant source intensity

308 function (f) is estimated using the kriging method. Structural parameters are determined by

309 minimizing the following objective function (Snodgrass and Kitanidis, 1997):

$$
L(\boldsymbol{\theta})=-\ln [p(\mathbf{z} \mid \boldsymbol{\theta})] \propto \frac{1}{2} \ln \left(|\Sigma| \cdot\left|\mathbf{X}^{T} \mathbf{H}^{T} \Sigma^{-1} \mathbf{H} \mathbf{X}\right|\right)+\frac{1}{2} \mathbf{z}^{T} \Xi \mathbf{z}
$$

310 in which:

$$
\begin{aligned}
& \Sigma=\mathbf{H Q H} \mathbf{H}^{T}+\mathbf{R} \\
& \Xi=\Sigma^{-1}-\Sigma^{-1} \mathbf{H X}\left(\mathbf{X}^{T} \mathbf{H}^{T} \Sigma^{-1} \mathbf{H X}\right)^{-1} \mathbf{X}^{T} \mathbf{H}^{T} \Sigma^{-1}
\end{aligned}
$$

311 the minimization of Equation (13) is a well-posed problem, since the number of observation $\mathbf{z}$

312 is greater than the number of structural parameters $\boldsymbol{\theta}$. In equations (14) and (15), $\mathbf{R}$ is the

313 covariance matrix of error in the observational data (v). It should be noticed that the value of

314 the unknown mean $\beta$ is not relevant as it does not appear in the Equations (13-(15). The $\beta$

315 coefficients are eliminated from Equation (13) by averaging over all possible values of it

316 (Hoeksema and Kitanidis, 1985, Kitanidis, 1995).

317 Once the structural parameters $\boldsymbol{\theta}$ are calculated, the intensity function is estimated through 318 a kriging system (De Marsily, 1986):

$$
\hat{\mathbf{f}}=\boldsymbol{\Lambda} \mathbf{z}
$$

319 Equation (16) is a linear estimator. It is unbiased and minimizes the estimate error variance

320 (Boano et al., 2005, Butera et al., 2013), in other words:

$$
\begin{gathered}
E[\hat{\mathbf{f}}-\mathbf{f}]=0 \\
\min _{\hat{\mathbf{f}}} E\left[(\hat{\mathbf{f}}-\mathbf{f})-(\hat{\mathbf{f}}-\mathbf{f})^{T}\right] .
\end{gathered}
$$


$321 \Lambda$ is a $[N \times M]$ matrix of Kriging weights that obtained from solving the following system of 322 equation:

$$
\left[\begin{array}{ll}
\boldsymbol{\Sigma} & \mathbf{H X} \\
(\mathbf{H X})^{T} & 0
\end{array}\right]\left[\begin{array}{l}
\mathbf{\Lambda}^{T} \\
\mathbf{M}
\end{array}\right]=\left[\begin{array}{c}
\mathbf{H Q} \\
\mathbf{X}^{T}
\end{array}\right]
$$

323 where $\mathrm{M}$ is a $[1 \times N]$ matrix of Lagrange multipliers (De Marsily, 1986). The mean of the

324 release history is then estimated by equation (16), while its covariance matrix $\mathbf{V}$ can be 325 evaluated as:

$$
\mathbf{V}=-\mathbf{X M}+\mathbf{Q}-\mathbf{Q} \mathbf{H}^{T} \boldsymbol{\Lambda}^{T}
$$

326 Using Equation (20) the confidence interval of 95\% can also be determined, so that for every instant of time $t_{i}$, the confidence interval can be calculated as $\hat{f}_{i} \pm 2 \sqrt{V_{i i}}$ in which $V_{i i}$ is the estimation error variance of $\hat{f}_{i}$.

The GS method is a practical and efficient method, but sometimes it obtains non-physical results, including negative concentrations. Usually, this problem is alleviated by introducing additional constraints to the unknown variable (Box and Cox, 1964, Snodgrass and Kitanidis, 1997, Michalak and Kitanidis, 2003, 2004a). This constrain is imposed by using a power transformation of the unknown variables. The new unknown function is written as follows:

$$
f=\alpha\left(\mathbf{f}^{1 / \alpha}-1\right)
$$

334 where $\alpha$ is a small positive parameter, the value of which is chosen in a way that ensure $f^{f / s}-\alpha$

335 . Kitanidis and Shen (1996) presented a method for choosing the optimal value of parameter $\alpha$ 336

337 Then, the transformed variable $\mathrm{f}^{/ /}$should be substituted to original variable $\mathrm{f}$ in equation (6), 338 so equation (6) is replaced by the following one:

$$
\mathbf{z}=\mathbf{h} \phi \mathbf{f})+\mathbf{v}
$$

339 in which:

$$
\mathbf{h}(\mathbf{f})=\mathbf{h}\left[((\mathbf{f} / \alpha) / \alpha)^{\alpha}\right]
$$


340 since the model is no longer linear with respect to the transformed variable $\mathbf{f}^{\text {/ }}$, the solution

341 must be evaluated using successive iterations. More details could be found in Kitanidis (1995).

342 The method can easily be extended to the case of $m$ multiple independent point sources located

343 at $\mathrm{x}=\left[x_{1}, x_{2}, \mathrm{~K}, x_{m}\right]$ and $\mathrm{p}$ distinct measurement points located at $\mathrm{x}_{o b s}=\left[x_{1}, x_{2}, \mathrm{~K}, x_{p}\right]$.

344 Regarding to the linearity of the ADE with respect to the concentration $\mathrm{C}(\mathrm{x}, \mathrm{t})$, it can be

345 written:

$$
C\left(x_{o b s}, t\right)=\sum_{j=1}^{m} f_{j}(\tau) K\left(x_{o b s_{i}}-x_{j}, t-\tau\right) \Delta \tau
$$

346 In which )24(is the number of observation points. The matrix form of equation $i=1,2, \ldots, p$

347 is as follows:

348 where:

$$
\mathbf{z}=\mathbf{H} \mathbf{f}+\mathbf{v}
$$

$$
\begin{aligned}
& \mathbf{z}^{T}=\left[\begin{array}{llll}
\mathbf{z}_{1} & \mathbf{z}_{2} & \mathrm{~K} & \mathbf{z}_{P}
\end{array}\right] \\
& \mathbf{f}^{T}=\left[\begin{array}{lll}
\mathbf{f}_{1} & \mathbf{f}_{2} & \mathrm{~K} \mathbf{f}_{m}
\end{array}\right] \\
& \mathbf{H}=\left[\begin{array}{ccc}
\mathbf{H}_{11} & \mathrm{~L} & \mathbf{H}_{1 m} \\
\mathrm{M} & \mathrm{O} & \mathrm{M} \\
\mathbf{H}_{i 1} & \mathrm{~L} & \mathbf{H}_{p m}
\end{array}\right]
\end{aligned}
$$

349 Equation (25) is a system of equation in which, $\mathrm{H}_{i j}, \mathrm{i}=1,2, \ldots, \mathrm{p}, \mathrm{j}=1,2, \ldots, \mathrm{m}$, is the transfer

350 matrix corresponding with the effect of the pollutant source release at $x_{i}$ on the measured

351 concentration data at $x_{j}$. Since pollutant sources are independent, the covariance matrix is a

352 block matrix as follows:

$$
\mathbf{Q}=\left[\begin{array}{llll}
\mathbf{Q}_{1} & \mathrm{~L} & 0 & 0 \\
0 & \mathbf{Q}_{2} & \mathrm{~L} & 0 \\
\mathrm{M} & \mathrm{M} & \mathrm{O} & \mathrm{M} \\
0 & 0 & \mathrm{~K} & \mathbf{Q}_{m}
\end{array}\right]
$$

353 The rest of steps for solving the system of equations (22) are similar to solving for a single

354 pollutant source, described in the previous sections. Figure 4 represents a flowchart of overall 355 identification process. 


\section{Results and Discussion}

357 In this section an application of the proposed method for simultaneous identification of 358 pollution source characteristics in a river network is presented. For this purpose, a hypothetical 359 river network consisting of a main stream (B1) and two tributaries (B2 and B3) with unsteady

360 flow conditions and irregular cross-sections has been considered. The general outline of the

361 considered river network along with the arrangement of main and secondary stations is shown

362 in Figure 5. The main and secondary stations were placed based on the criteria mentioned in

363 section 2.2. First, by assuming the activity duration of 10 hours and more for retrieval and

364 based on the calculated travel time from Equation (4), the location of main stations in all

365 branches, was determined. After that, based on the desired accuracy for spatial range,

366 secondary stations were located in the intervals of 8, 7 and $9 \mathrm{~km}$ for the main, second and third

367 branches, respectively.

368 A complete list of main and secondary stations of each branch, along with its distance from

369 the upstream and the travel time between two successive main stations, is given in Table 1. It

370 can been seen from Table 1 that the travel time between two successive main stations is always

371 less than or equal to the expected activity time for retrieval (10 hours). It also should be

372 mentioned that main stations which located at the beginning of branches, namely M1, M5 and

373 M7, are only used to record upstream boundary conditions of the forward flow and transport model and are not used in the identification process. 


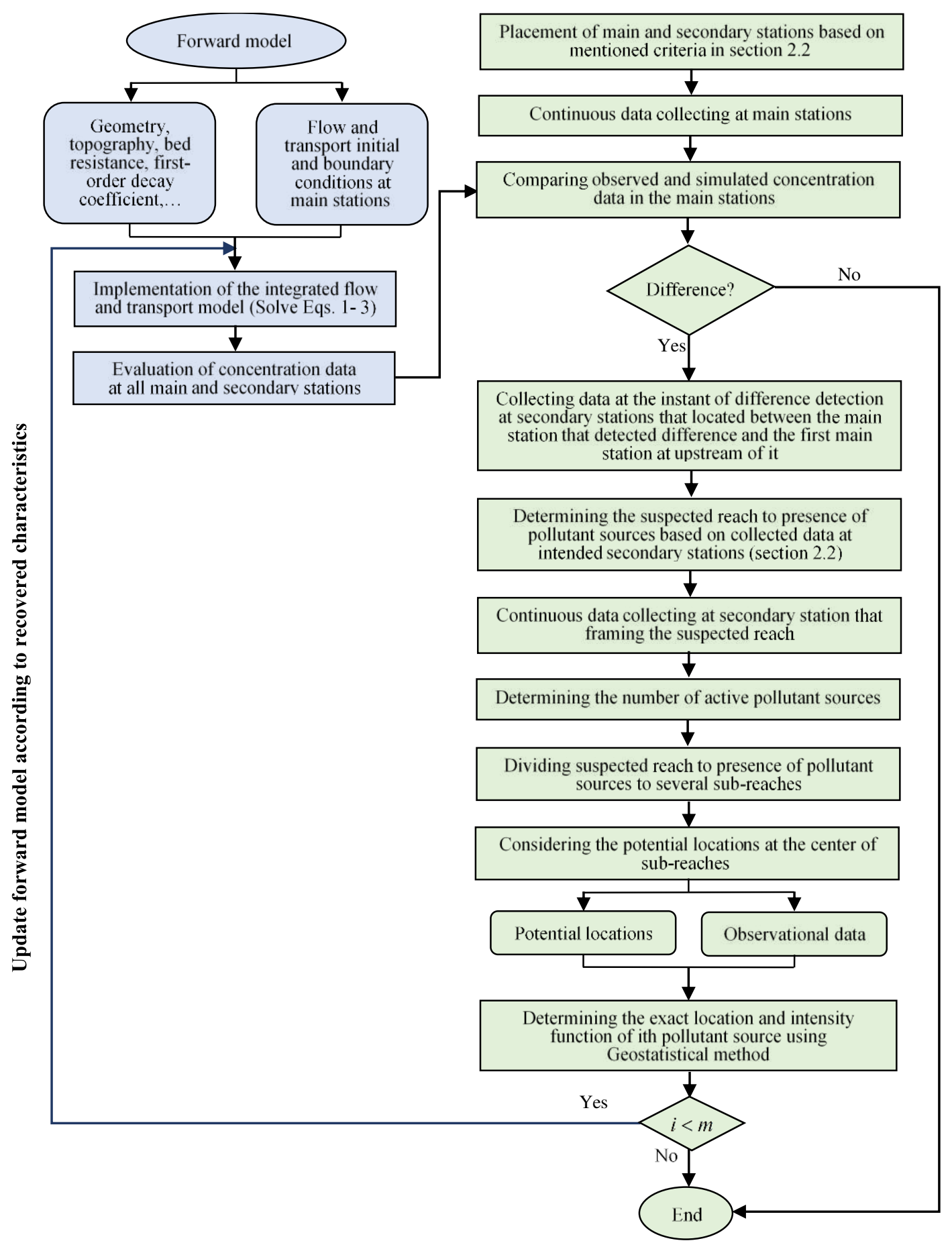

Figure 4- Flowchart of identification process

377 After placement of main and secondary stations, in order to calculate the spatial and 378 temporal distribution of pollutant concentration in all stations, the forward flow and transport 379 model are performed twice with give boundary condition (Figure 6). First without considering 380 pollutant sources and then with considering them. The first set of results are used as simulated 381 data and the second ones are used as observed data. In order to evaluate the performance of 
382 proposed method three different scenarios in terms of number, release location and activity

383 duration has been considered. The main characteristics of those scenarios were listed in Table

384 2. Complementary explanations for each scenario are given below.



385

386

387

Figure 5- The schematic of hypothetical river network along with the arrangement of the main and secondary stations

Table 1- Monitoring stations

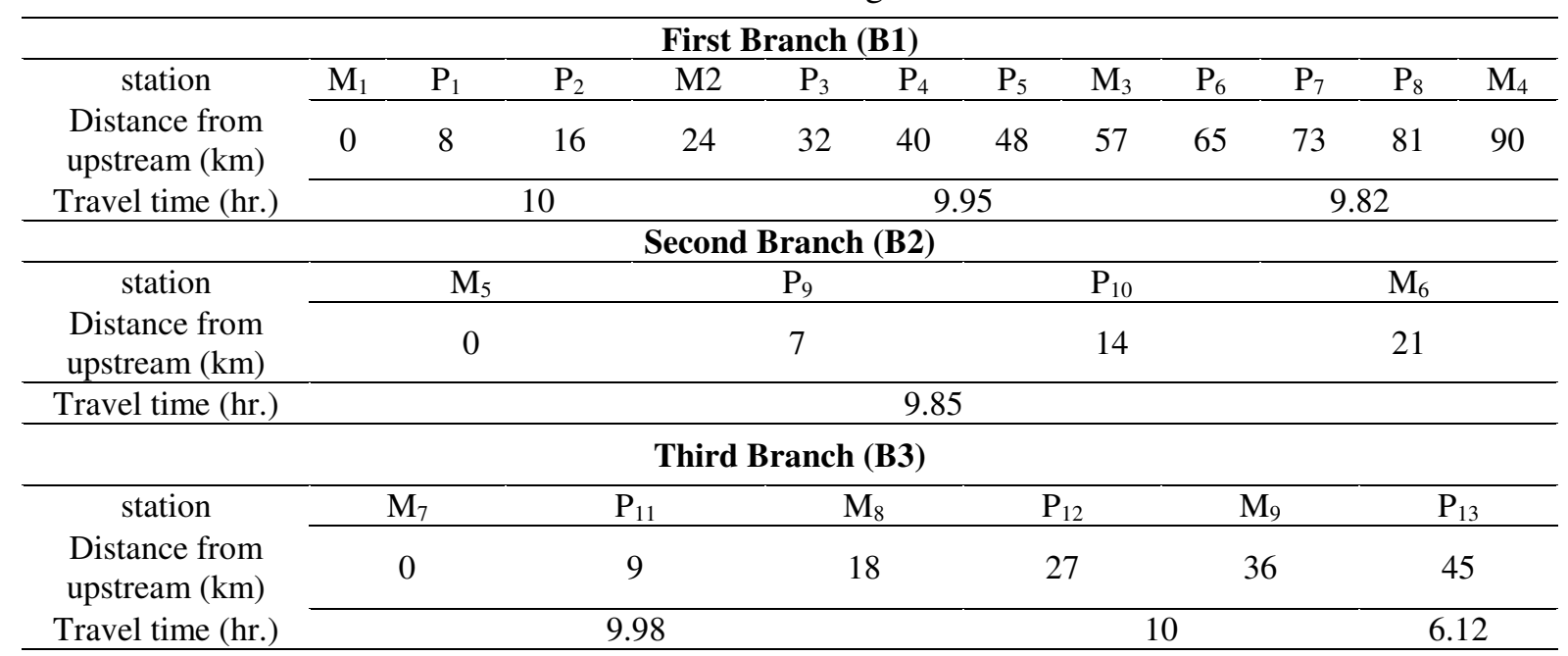



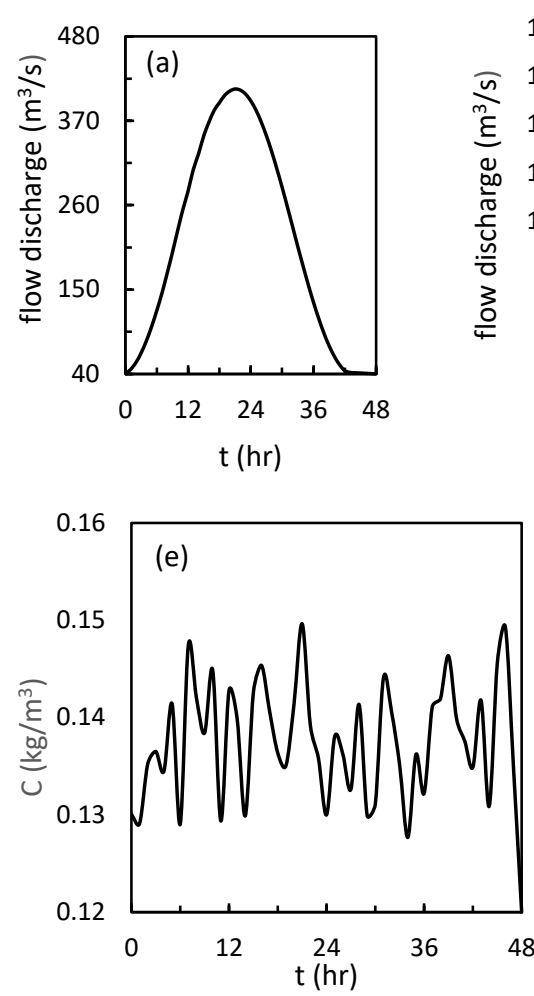
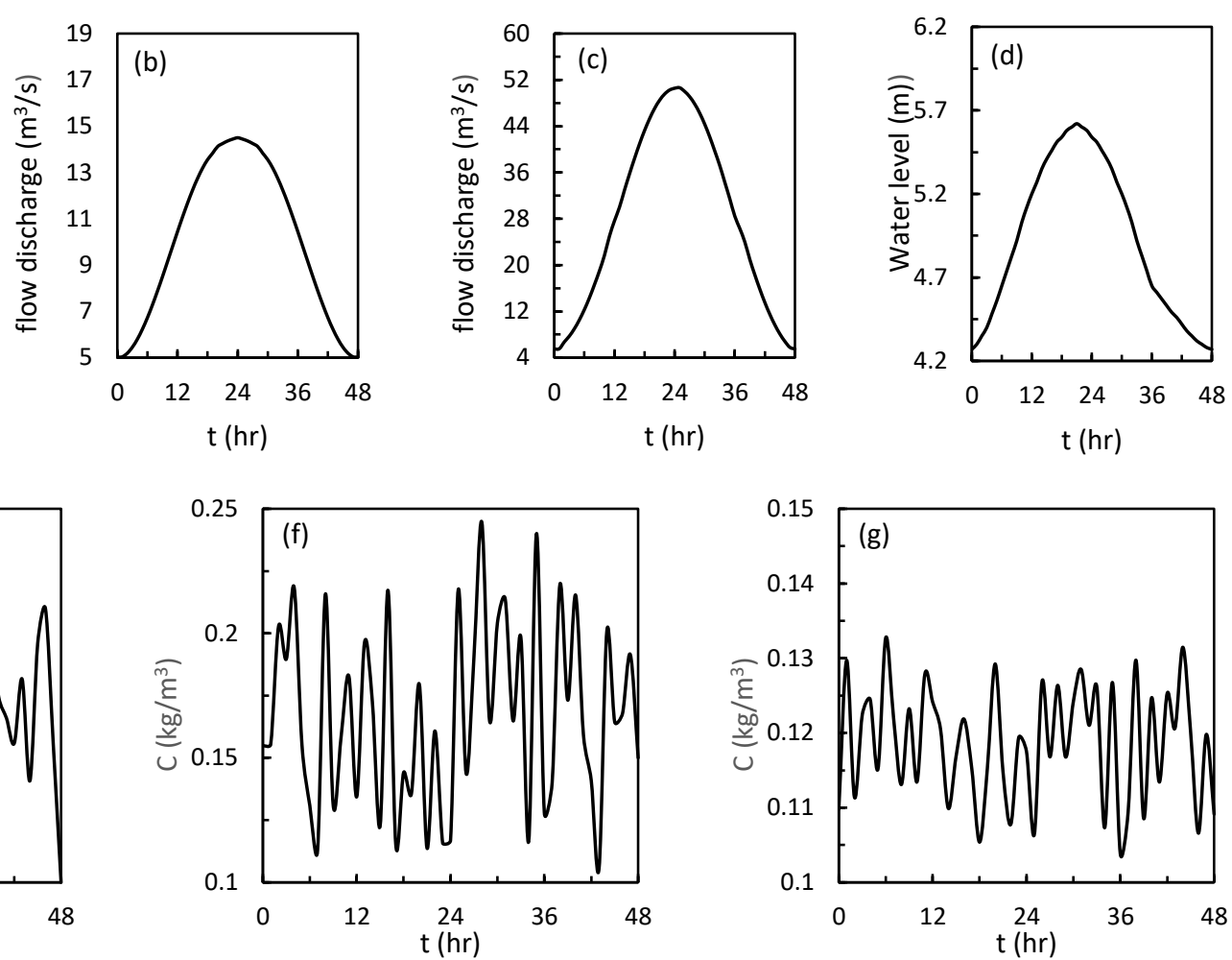

Figure 6- Flow and transport boundary conditions, (a)-(c) upstream boundary conditions of flow model at M1, M5 and M7, respectively, (d) upstream boundary conditions of flow model at M4 and (e)-(g) upstream boundary conditions of transport model at M1, M5 and M7, respectively.

Table 2- The main characteristics of different considered scenarios

\begin{tabular}{|c|c|c|c|c|}
\hline Scenario & $\begin{array}{l}\text { Number of Active } \\
\text { Sources }\end{array}$ & $\begin{array}{c}\text { Release location (Branch/Distance from } \\
\text { Upstream) }\end{array}$ & $\begin{array}{l}\text { Activity Duration } \\
\text { (hr.) }\end{array}$ & $\begin{array}{c}\text { Simultaneous } \\
\text { Active } \\
\text { Sources }\end{array}$ \\
\hline 1 & 1 & B2-8.75 & 11 & - \\
\hline \multirow{2}{*}{2} & \multirow{2}{*}{2} & B2-8.75 & 11 & \multirow{2}{*}{ No } \\
\hline & & B1-46 & 16 & \\
\hline \multirow{3}{*}{ 3- Test 1} & \multirow{3}{*}{3} & B2-8.75 & 11 & \multirow{3}{*}{ Yes } \\
\hline & & B1-46 & 16 & \\
\hline & & B1-10 & 13.5 & \\
\hline \multirow{3}{*}{3 -Test 2} & \multirow{3}{*}{3} & B2-8.75 & 11 & \multirow{3}{*}{ Yes } \\
\hline & & B1-10 & 13.5 & \\
\hline & & B1-46 & 16 & \\
\hline
\end{tabular}

\subsection{Scenario 1: one active source}

394 In this example, it is assumed that there is only one active pollution source at $8.75 \mathrm{~km}$ of the upstream end of the second branch (B2) with activity duration of 11 hours (Figure 7). As mentioned in Section 2.2, it is necessary to first determine the suspected reach to presence of

397 the source by comparing the observed and simulated concentration data at all main stations.

398 Figure 8 shows a comparison of observed and simulated concentration data at main stations for

399 this example. It should be noted that the initial period with zero concentration is due to the 
initial condition that was chosen for the sake of simplicity, and it does not affect the results in other ways. It can be seen from Figure 8 that the first main station which recorded the difference end of the second branch of the hypothetical river network.
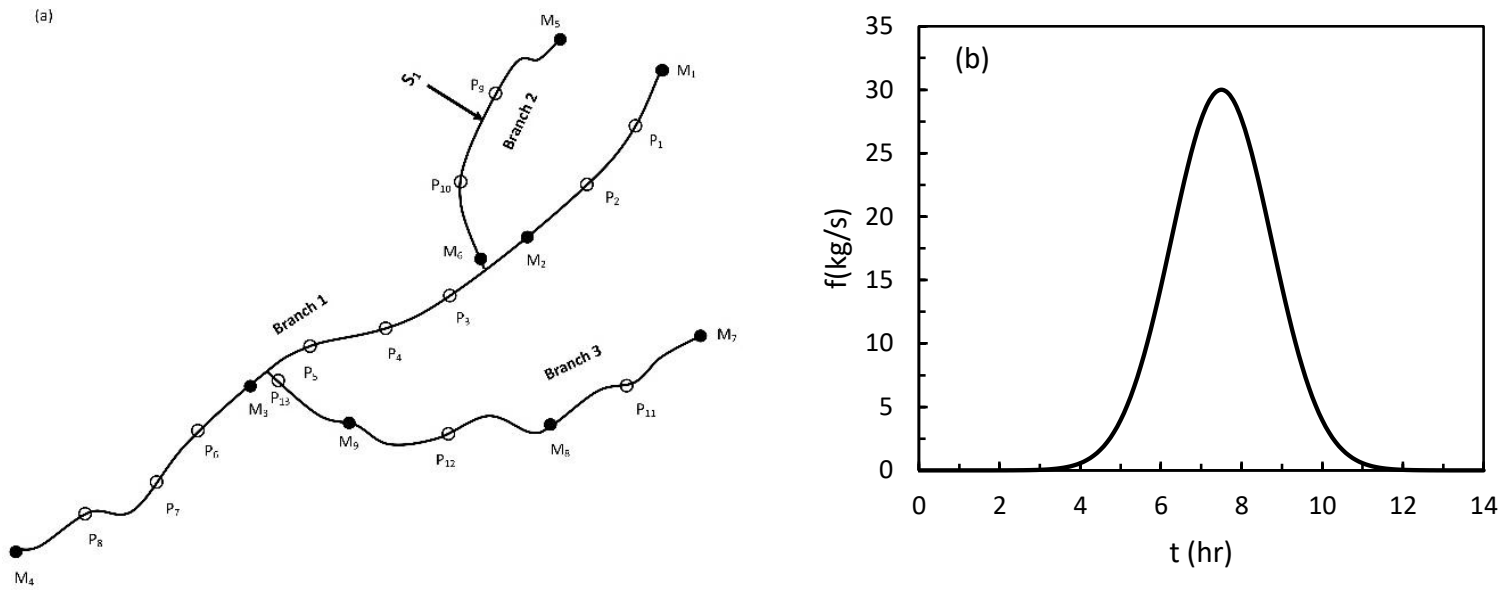

Figure 7- (a) location and (b) intensity function of pollutant source (scenario 1)
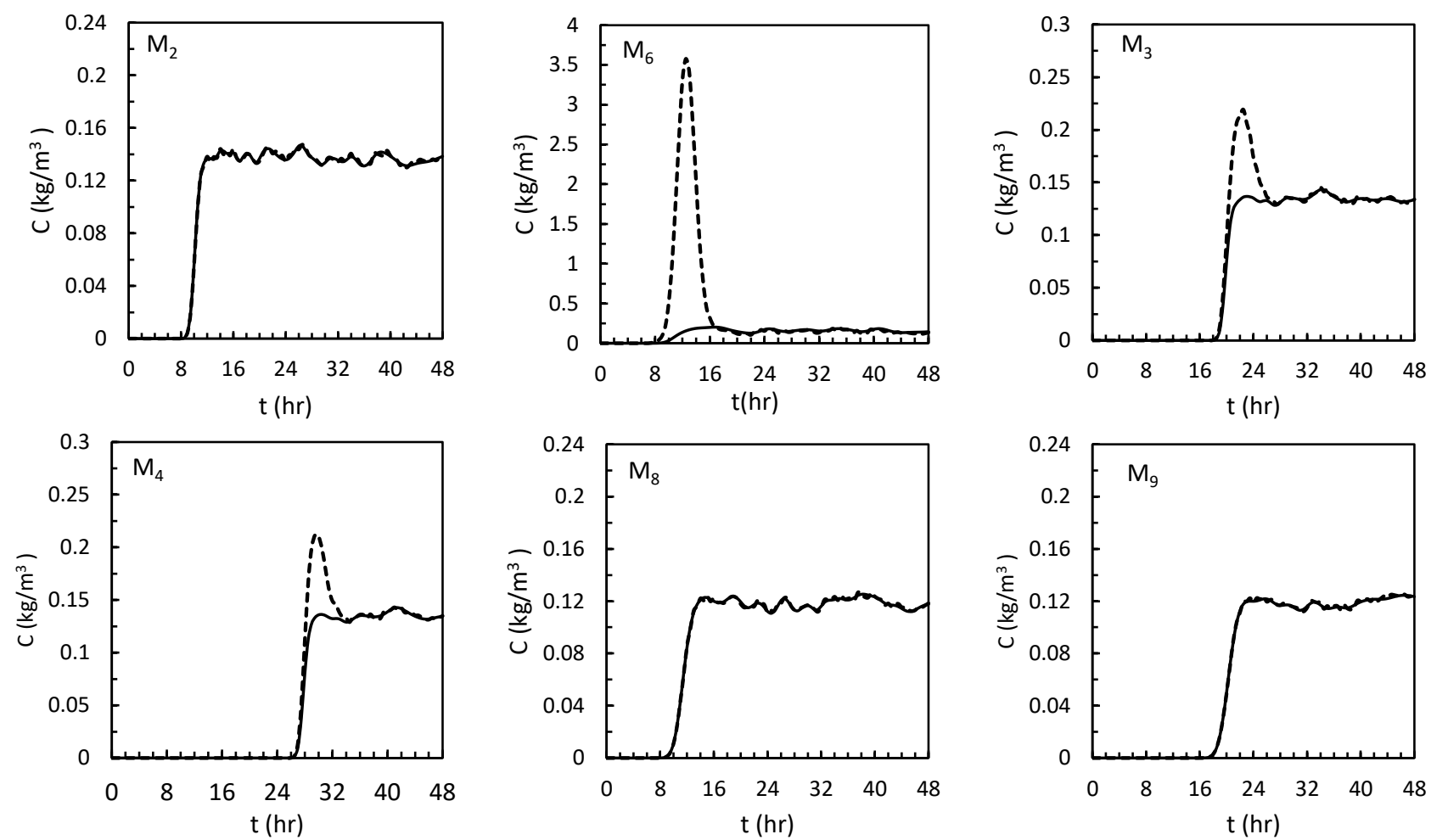

Figure 8- Comparison of simulated and observed data in main stations (scenario 1) (solid line: simulated and dashed line: observed data)

After detection the difference in data at M6, a concentration observation data should be

collected at all secondary stations that located at upstream of M6 (namely, P9 and P10) at the instant of difference detection. Figure 9 shows the collected data at these secondary stations 
410 and their comparison with the simulated data. As it can be seen from Figure 9, there is a

411 significant difference between the observed and simulated concentration data at P10, while the

412 observed and simulated data at P9 are exactly the same. Therefore, it can be concluded that the

413 release point of the pollutant source is in the reach between P9 and P10, i.e. at the range of 7

414 to $14 \mathrm{~km}$ of the upstream end of the B2.

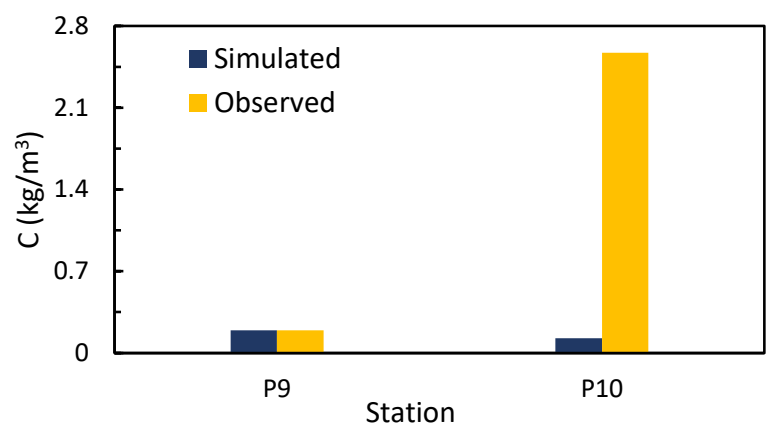

Figure 9- Comparison of simulated and observed data in secondary stations at the instant of difference detection between the simulated and observed data in M6 (scenario 1)

Subsequently, secondary stations that located at the upstream and downstream of suspected reach, should begin to permanent data collection to ensure that there is no other active source. Figure 10 shows a comparison of observed and simulated data at P9 and P10. As it can be seen from Figure 10, there is no difference between the observed and simulated data at P9, which means that there is no active source at the upstream of suspected reach, during the period of activity of the discovered source. Comparison of these two series of data in P10 shows the difference. According to the general form and peak concentration of observed concentrationtime curve and comparing it with the concentration-time curve at M6 (Figure 8), it can be deduced that this difference caused by the source that just has been discovered and there are no other active sources.

In the next step, the suspected reach to presence of pollutant source (i.e., 7 to $14 \mathrm{~km}$ of the upstream end of the B2) is divided into two sub-reaches (namely from 7 to $10.5 \mathrm{~km}$ and 10.5 to $14 \mathrm{~km}$ ) and the potential locations of the pollutant source are considered at the center of these sub-reaches (i.e., 8.75 and $12.25 \mathrm{~km}$ of the upstream end of the B2). Then, by implementing the inverse model and using the spatial distribution of concentration data at all 
432 stations located downstream of suspected reach and at the instant of full passage of pollution

433 cloud from P10, exact location and approximate intensity function of pollutant source are

434 determined. Figure 11 shows the exact and recovered intensity function of the pollutant source

435 with 95 percent confidence interval for both potential locations. As it can be seen from the

436 Figure 11, for the case where the potential location is equal to the exact location of the assumed

437 pollutant source (i.e. $8.75 \mathrm{~km}$ of the upstream end of the B2), there is a good match between

438 the exact and recovered intensity function. While, in the case where the potential location is

439 considered at $12.25 \mathrm{~km}$ of the upstream end of the B2, a close to zero amount for intensity

440 function has been obtained.
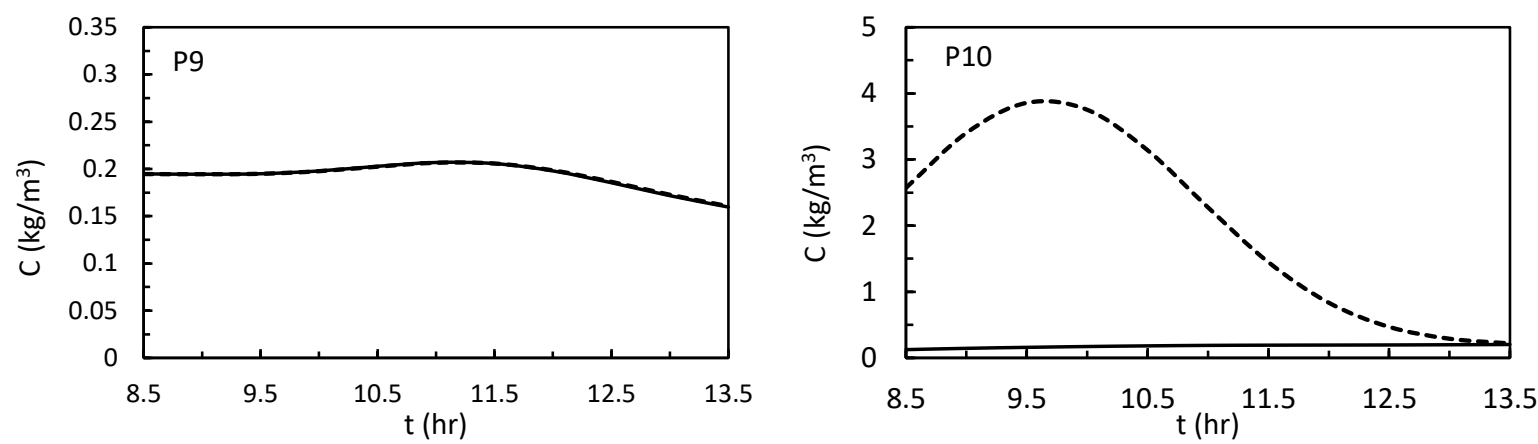

441

442

443

Figure 10- Comparison of observed and simulated data in secondary stations located at the upstream and downstream of suspected reach during the period of permanent data collection by those stations (scenario 1) (solid line: simulated and dashed line: observed data)
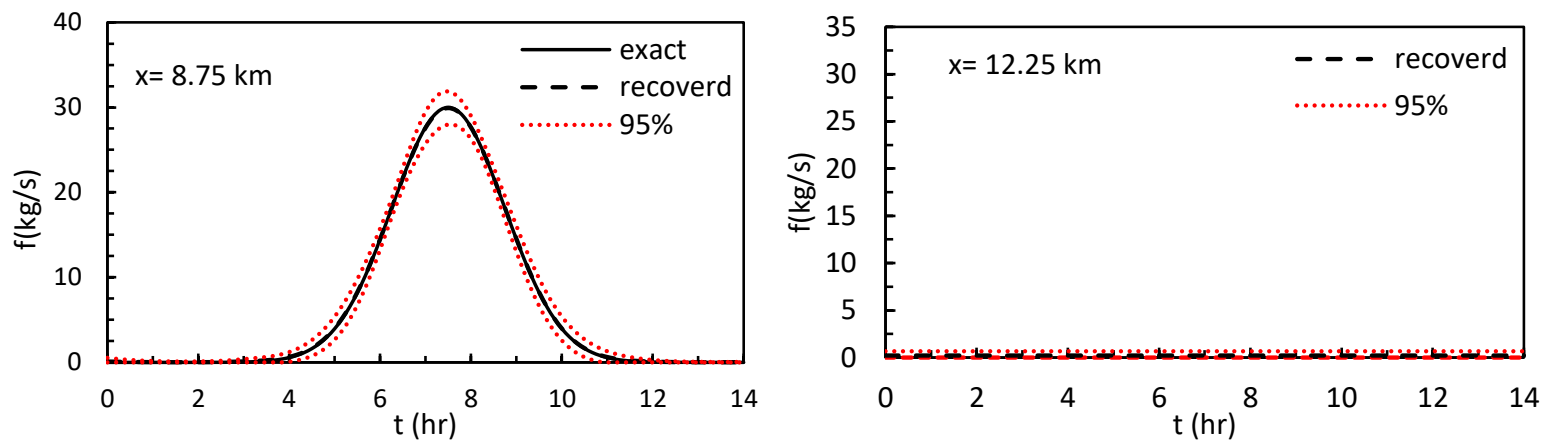

444

445

446

447

448

449

Figure 11- Recovered intensity function at two potential locations using observed data at all main and secondary stations that located downstream of the suspected reach (scenario 1)

After determining the exact location of the source, it is necessary to identify its intensity function more accurately, assuming that the source location is known. So, the intensity function is recovered using observed time-concentration data at the first main station at downstream (M6). The results are shown in Figure 12. The error indices for both approximate recovery 
450 using the spatial distribution of concentration data and the exact recovery using time451 concentration data are given in Table 3. It should be mentioned that, the indices that used to 452 evaluate the performance of proposed method include square of correlation coefficient $\left(\mathrm{R}^{2}\right)$, 453 root mean square error (RMSE), mean absolute error (MAE) and Euclidean distance (de). The 454 last one, indicates the distance between the upper $(\sigma u)$ and lower $(\sigma l)$ bound of the $95 \%$ 455 confidence interval and it is used to evaluate the uncertainty of recovered release history based 456 on the observation data (Equation (30))

$$
d e=\sqrt{\sum_{i=1}^{n}\left(\sigma u_{i}-\sigma l_{i}\right)^{2}}
$$

Figure 12 and the error indices in Table 3 indicate that in both cases the proposed model has been retrieved the intensity function with almost a same accuracy. The only difference is concerned with the width of $95 \%$ confidence interval, which is wider in the case of retrieval using spatial distribution of concentration data. This means that there are more release histories that consistent with the observations. This is also an indication of the increased uncertainty in estimations. The main reason for this results is sparsity of spatial distribution of concentration data compared to time-concentration data, which makes the ill-posedness issue more sever and causes more uncertainty in identification process.

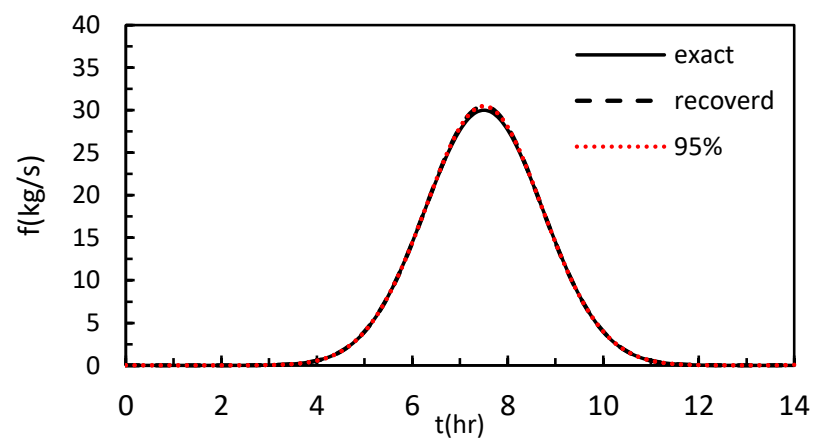

Figure 12- Recovered intensity function by considering the exact location of the source and using observed concentration-time data at the first main station at downstream (M6) 
Table 3- Error indices of scenario 1

\begin{tabular}{ccc}
\hline Index & $\begin{array}{c}\text { Recovery using spatial distribution } \\
\text { of concentration data }\end{array}$ & $\begin{array}{c}\text { Recovery using observed concentration-time } \\
\text { data at the first main station at downstream } \\
(\mathrm{M} 6)\end{array}$ \\
\hline $\mathrm{R}^{2}(\%)$ & 99.99 & 99.99 \\
$\mathrm{RMSE}(\mathrm{kg} / \mathrm{s})$ & 0.05 & 0.04 \\
$\mathrm{MAE}(\mathrm{kg} / \mathrm{s})$ & 0.045 & 0.027 \\
$\mathrm{de}(\mathrm{kg} / \mathrm{s})$ & 9.12 & 0.24 \\
\hline
\end{tabular}

\section{3.2. Scenario 2: two asynchronous active sources}

473 In this example, it was assumed that there are two active pollutant sources in the river network

474 during the simulation time, so that the start time of activity of the second source is after the end 475 of activity of the first pollutant source. The first source was considered similar to the scenario 476 one, at $8.75 \mathrm{~km}$ of the upstream end of the B2 and the second source assumed at $46 \mathrm{~km}$ of the 477 upstream end of the B1 (Figure 13). After identification of the first source, similar to the 478 scenario 1 , the forward model is modified considering the identified location and release 479 history of the first source. After revising the forward model, a comparison of the observed and 480 simulated data at the main stations (Figure 14) shows that a difference between these series of 481 data at the M3 located at $57 \mathrm{~km}$ of the upstream end of the B1. This indicates the presence of an active source at the upstream of that station. So, it is necessary to collect a concentration data at the instant of recording the difference at all secondary stations located between station M3 and the first main stations upstream (i.e. M2, M6 and M8). Figure 15 depicts the collected data at these secondary stations and their comparison with the simulated data. As can be seen

486 from Figure 15 the only secondary station that recorded the difference between the observed 487 and simulated data is the P5, located $48 \mathrm{~km}$ of the upstream end of the B1.Hence, it can be said that the suspected reach to presence the second source is between P5 and the upstream secondary station (P4). 



Figure 13- (a) location and (b) intensity function of pollutant sources (scenario 2)
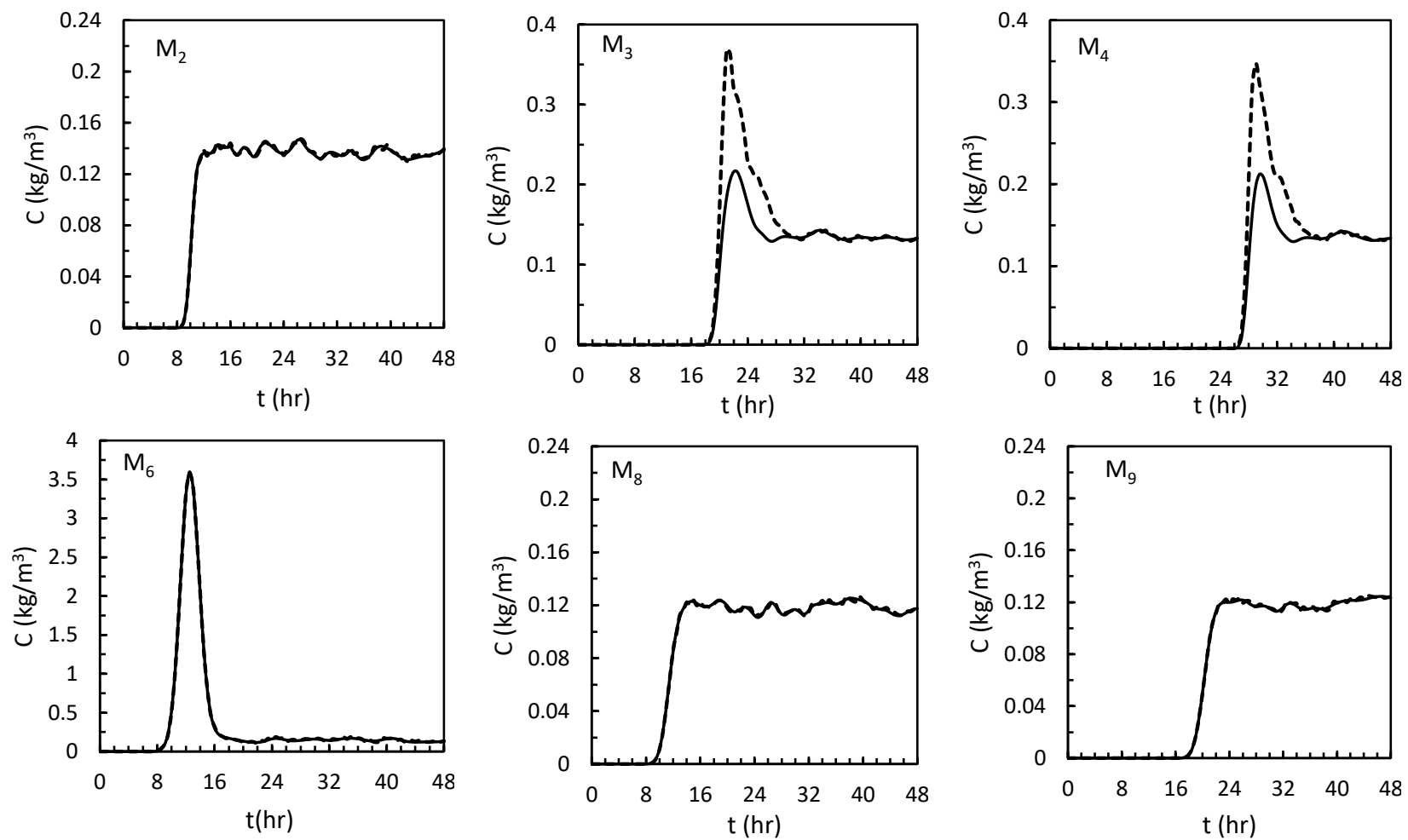

491

492

Figure 14- Comparison of simulated and observed data in main stations (scenario 2) (solid line: simulated and dashed line: observed data)

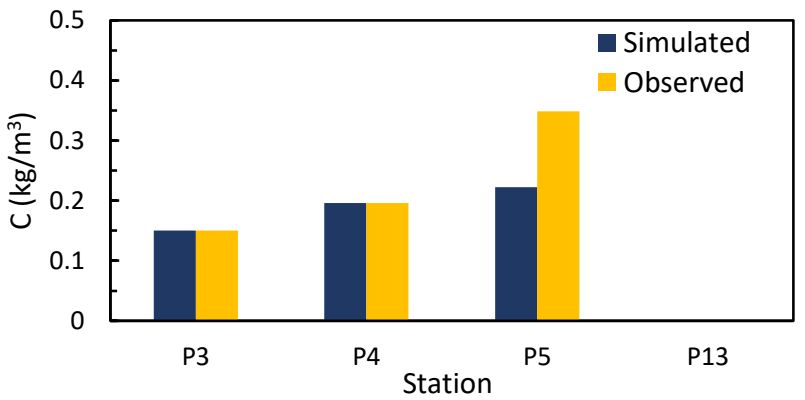

Figure 15- Comparison of simulated and observed data in secondary stations at the instant of difference detection between the simulated and observed data in M3 (scenario 2) 

of suspected reach, should begin to permanent data collection to ensure that there is no other

497 active source. A comparison of observed and simulated data at P4 and P5 are shown in Figure

498 16. As can be seen from Figure 16, there is no difference between the observed and simulated 499 data at P4, which means there is no active source at the upstream during the activity time of 500 the discovered source. A comparison of these two sets of data in the P5 represents a difference.

501 By comparing the general form and peak concentration of observed concentration-time curve and concentration-time curve at M3 (Figure 14), it can be concluded that this difference is due 503 to the discovered source and there are no other active pollutant sources.
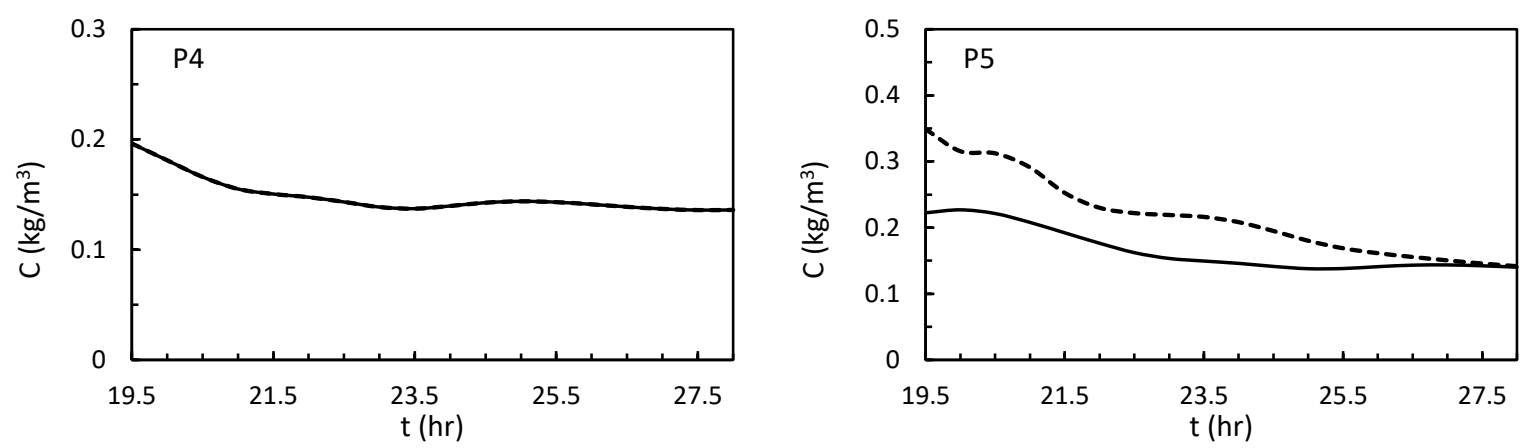

504

505

506

507

508

509

510

511

512

513

514

Figure 16- Comparison of observed and simulated data in secondary stations located at the upstream and downstream of suspected reach during the period of permanent data collection by those stations (scenario 2) (solid line: simulated and dashed line: observed data)

In the next step, the suspected reach is divided into two sub-reaches with equal length and the potential locations of the source are considered in the center of each of these sub-reaches, namely 42 and $46 \mathrm{~km}$ of the upstream end of the B1. Then, the exact location of S2 and its approximate intensity function are determined by implementing inverse model and using the spatial distribution of concentration data in all stations located at the downstream of suspected reach. The results are presented in Figure 17. According to these results, it can be concluded that the second source is located $46 \mathrm{~km}$ of the upstream end of the B1, which is corresponded to the assumed location. 

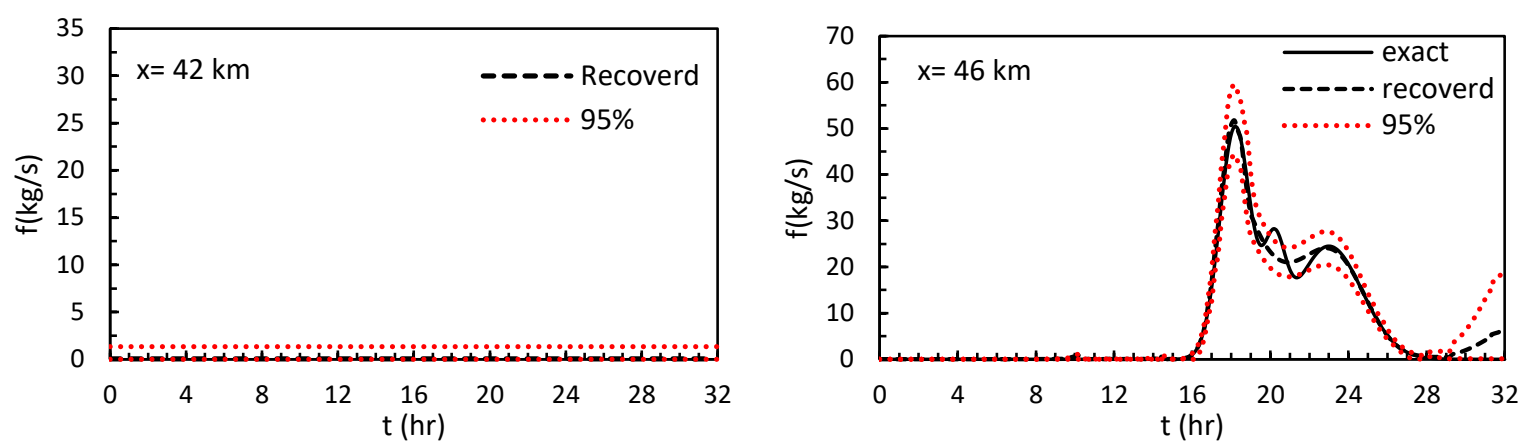

515

516

517

518

519

520

521

522

523

524

525

526

527

528

Figure 17- Recovered intensity function at two potential locations using observed data at all main and secondary stations that located downstream of the suspected reach (scenario 2)

Finally, by assuming the known source location and using concentration-time data at M3,

the intensity function is determined more accurately. The results are shown in Figure 18. The error indices for both approximate recovery using the spatial distribution of concentration data and the exact recovery using time-concentration data are given in Table 4. Figure 18 and the error indices in Table 4, suggested that the accuracy of the results obtained using the concentration-time data is slightly better than the accuracy of the results obtained using the spatial distribution of concentration data. In addition, the $95 \%$ confidence interval opening is wider at the case of recovery with spatial distribution of concentration data, which is interpreted as more uncertainty in results. Given that the spatial distribution of concentration data are usually sparse and the number of available data is much less than the desired temporal instants for retrieval of intensity function, the existence of a higher degree of uncertainty in the results is inevitable.

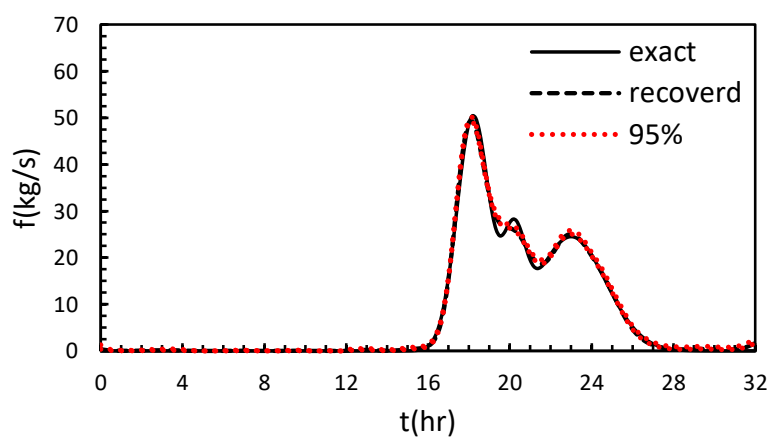

Figure 18- Recovered intensity function by considering the exact location of the source and using observed concentration-time data at the first main station at downstream (M3) 
Table 4- Error indices of scenario 2

\begin{tabular}{ccc}
\hline Index & $\begin{array}{c}\text { Recovery using spatial distribution } \\
\text { of concentration data }\end{array}$ & $\begin{array}{c}\text { Recovery using observed concentration-time } \\
\text { data at the first main station at downstream } \\
(\mathrm{M} 3)\end{array}$ \\
\hline $\mathrm{R}^{2}(\%)$ & 98.32 & 99.68 \\
$\mathrm{RMSE}(\mathrm{kg} / \mathrm{s})$ & 1.64 & 0.6975 \\
$\mathrm{MAE}(\mathrm{kg} / \mathrm{s})$ & 0.7996 & 0.3823 \\
$\mathrm{de}(\mathrm{kg} / \mathrm{s})$ & 81.2366 & 15.2694 \\
\hline
\end{tabular}

\subsection{Scenario 3: three active sources, with at least two simultaneously active}

536 In order to show the capabilities of the proposed model in the case where several sources are

537 simultaneously active, this example considered the identification of three sources that a part of the activity time of two of those sources coincide. The first source similar to the scenario 1 has been considered at $8.75 \mathrm{~km}$ of the upstream end of the B2 and the other two sources considered at 10 and $46 \mathrm{~km}$ of the upstream end of the B1. It is also assumed that the activity time of the

541 last two sources is after the end of the activity of the first source. In addition, it assumed that part of the activity time of the sources that located at 10 and $46 \mathrm{~km}$ of the upstream end of the B1 is simultaneous. This example is presented for two different cases in terms of the start activity time of pollutant sources. Complementary explanations for each case are given below.

\section{5 a) Test 1}

546 In the first case, it is assumed that the source at $46 \mathrm{~km}$ of the upstream end of the B1 starts its

547 activity earlier than the source at $10 \mathrm{~km}$ of the upstream end of the B1(Figure 19). After 548 identification of the first source, similar to what described in the scenario 1, the forward model 549 is modified according to recovered source characteristics. After revising the forward model, a 550 comparison of observed and simulated data at the main stations (Figure 20), first shows a difference between these two set of data at the M3 located at $57 \mathrm{~km}$ of the upstream end of the B1. A few hours later, while the pollution cloud has not yet completely passed the M3, a

553 difference between the observed and simulated data at the M2 at $24 \mathrm{~km}$ of the upstream end of 554 the B1, is recognized. This means two sources are simultaneously active at upstream of these 
two main stations. In order to correctly identify the suspect reaches to presence of these two sources, it is necessary to collect a concentration data at the instant of difference detection at

557 all secondary stations between station M3 and M2 and the first main stations that located at 558 upstream of them. Figure 21 (a) and (b) represent a comparison of observed and simulated data 559 at sought secondary stations and at the instant of difference detection in M3 and M2, 560 respectively.


Figure 19- (a) location and (b) intensity function of pollutant sources (scenario 3-test 1)
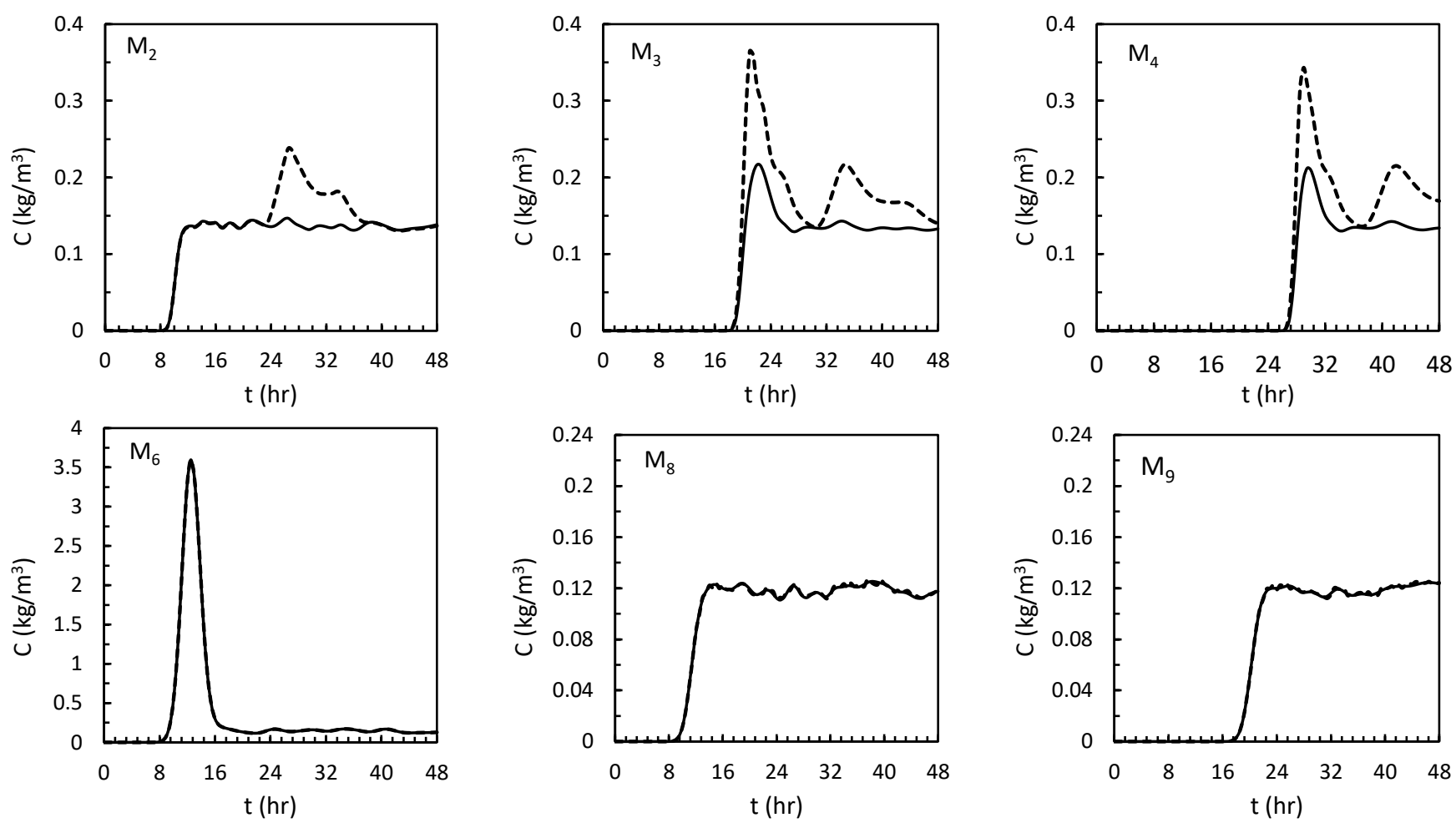

562 Figure 20- Comparison of simulated and observed data in main stations (scenario 3-test1) (solid line: simulated and dashed line: observed data) 

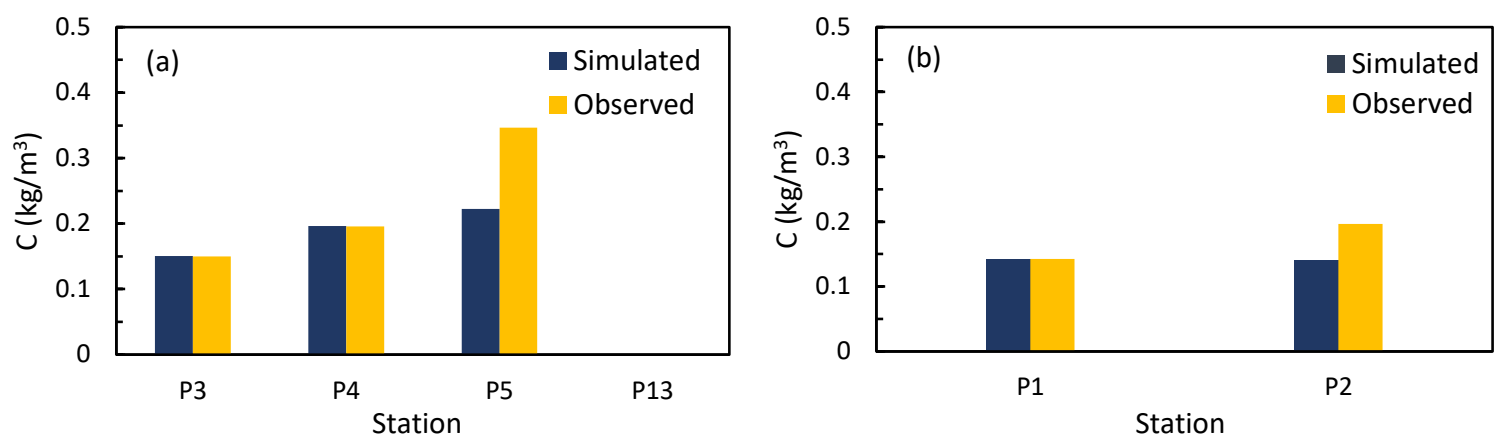

564

565

566

567

568

569

570

571

572

573

574

575

576

577

578

579

580

581

582

583

584

585

Figure 21- Comparison of simulated and observed data in secondary stations at the instant of difference detection between the simulated and observed data in (a) M3 and (b) M2 (scenario 3-test1)

In addition, to ensure that there are no other active sources, the concentration-time data that has been collected at secondary stations at the upstream and downstream of suspected reaches are compared with simulated data. It should be noted that the beginning instant of data collection is from the instant of difference detection at M3 and M2. Figure 22 shows a comparison of observed and simulated data at secondary stations P1, P2, P4 and P5. As can be seen from Figure 22, there is no difference between observed and simulated data at P1, which means that there is no active source at upstream of that station during the activity of detected source. A comparison of these two sets of data in $\mathrm{P} 2$ shows a difference. By comparing the general form and peak concentration of C-t curve with C-t curve at M2 (Figure 20), it can be deduced that this difference is due to the discovered source and there is no other active source. Observed and simulated data at P4 and P5 also show difference. By a similar argument, it can be concluded that this difference is due to the discovered sources and that there is no other active source at upstream of these stations.

After determining the suspected reaches to presence of two sources, their exact location and approximate intensity function are recovered using the spatial distribution of concentration data in all downstream stations. Given that the source which located at 40 to $46 \mathrm{~km}$ of the upstream end of the B1 has started its activity earlier, its exact location must be determined first. It should be noted that this case is fundamentally different from the two previous two scenarios. In the two previous scenarios, the spatial distribution of concentration data which used to determine the exact location and approximate intensity function had been collected at the 
instant of full passage of the pollution cloud from downstream secondary station. However, in

587 this test, due to the simultaneous activity of two pollutant sources, the exact location and

588 intensity function of the second pollutant source are determined using the spatial distribution

589 of concentration data at the instant of discovering the effect of third source. This is because the

590 observed data at the instant the full passage of pollutant cloud from the downstream secondary

591 station represented the combined effects of two sources, and therefore using of them may lead

592 to incorrect identification results. While at the instant of detection third source, its effect has

593 not yet reached the downstream, and the data that has been recorded at downstream main and

594 secondary stations shows only the effect of second source.
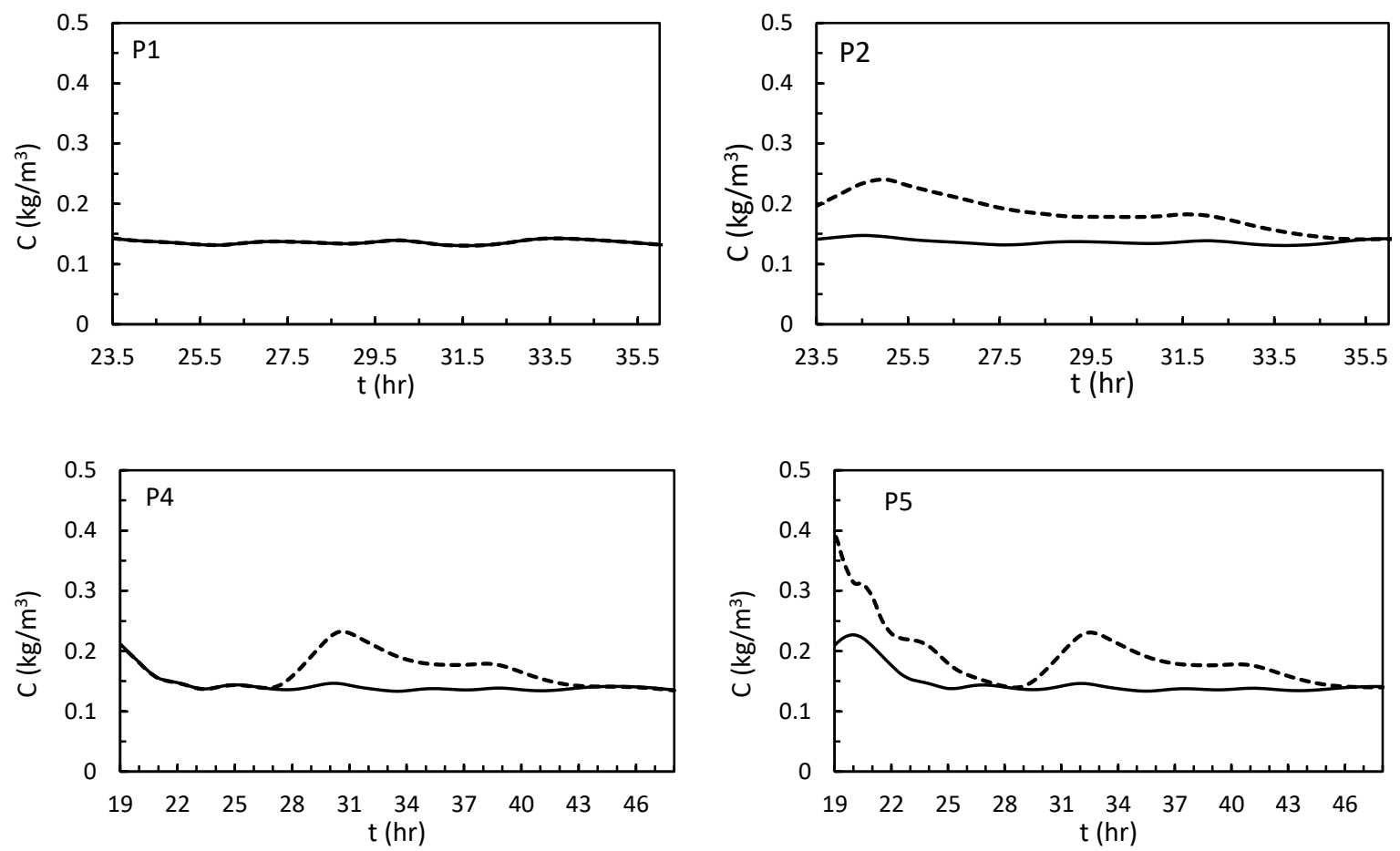

Figure 22- Comparison of observed and simulated data at secondary stations located at the upstream and downstream of suspected reaches during the period of permanent data collection by those stations (scenario 3-test 1) (solid line: simulated and dashed line: observed data)

The identification process is started by dividing the suspected reaches to presence of second

and third sources into two equal length sub-reaches. Then, potential locations of the pollutant sources are considered in the center of those sub-reaches and by implementing the inverse model the exact location and approximate intensity function of each source is determined.

602 Figure 23 shows the results of inverse model implementation for two potential locations for 
603 second source, i.e. 42 and $46 \mathrm{~km}$ of the upstream end of the B1. As can be seen from it, a close

604 to zero and a non-zero intensity functions have been obtained for 42 and $46 \mathrm{~km}$ potential

605 locations, respectively. Therefore, it can be concluded that the second source of is located at

$60646 \mathrm{~km}$ of the upstream end of the B1, which corresponds to the assumed location. Subsequently,

607 the location of third source is also determined using the spatial distribution of concentration

608 data at the instant that pollutant cloud fully passes from P2. The results of the inverse model

609 implementation for the two potential locations, i.e. 10 and $14 \mathrm{~km}$ of the upstream end of the

610 B1, are shown in Figure 24. As indicated in this figure, a non-zero intensity function is obtained

611 for the potential location of $10 \mathrm{~km}$. So, it can be concluded that the third source is released at

$61210 \mathrm{~km}$ of the upstream end of the B1, which corresponds to the assumed location.
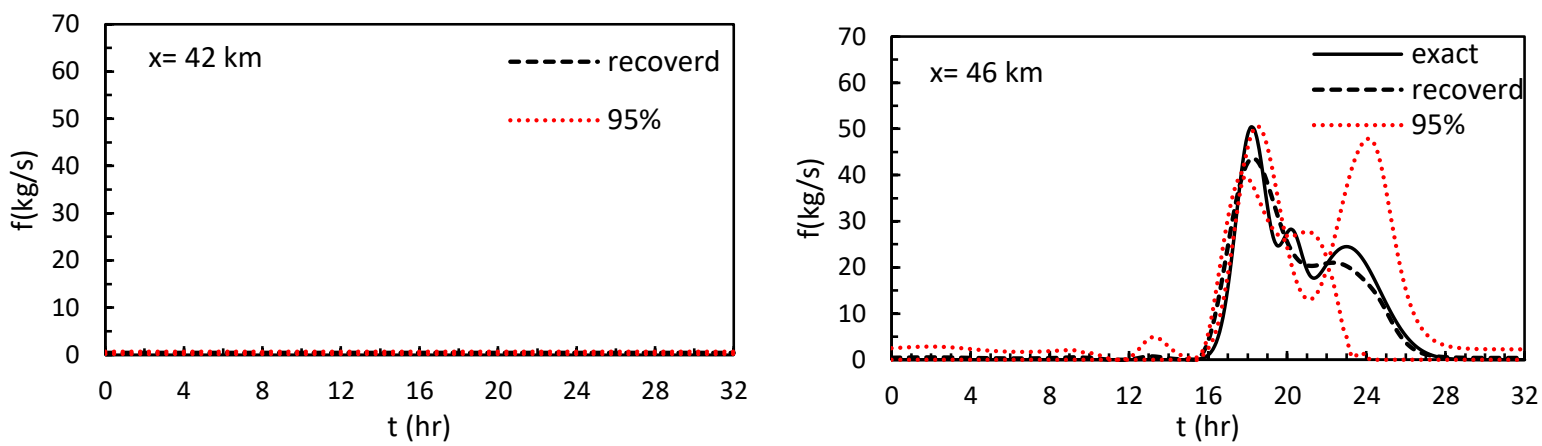

613 Figure 23- Recovered intensity function of $\mathrm{S}_{2}$ at two potential locations using observed data at all

614 main and secondary stations that located downstream of the suspected reach at the instant of recording

615
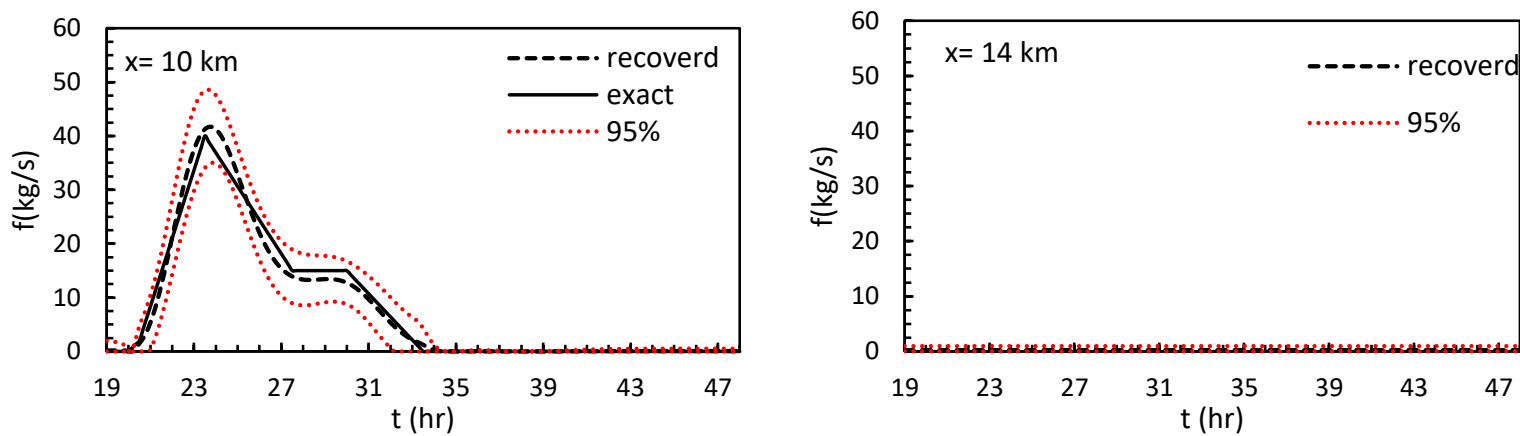

Figure 24- Recovered intensity function of $S_{3}$ at two potential locations using observed data at all main and secondary stations that located downstream of the suspected reach at the instant that the pollution cloud completely passes the $\mathrm{P} 2$ (scenario 3-test1)

Once the location of pollutant sources has been determined, their intensity functions should

620 be recovered more accurately, assuming known source locations. Due to the simultaneous

621 activity of two pollutant sources in this case, by starting from upstream, at first the exact 
622 intensity function of the third pollutant source (located $10 \mathrm{~km}$ from upstream of B1) is retrieved 623 using the concentration time data at M2. Then the forward model is modified, considering the 624 obtained characteristics of this source. Thus, the C-t observed data at M3 will only include the 625 effect of the second pollutant source ((located $46 \mathrm{~km}$ from upstream of B1)), and the exact 626 intensity function of this source can also be calculated.

627 The results of the recovery of the third source intensity function using the C-t observed data 628 at M2 are shown in Figure 25. Figure 26 shows the results of exact recovery of the intensity 629 function of second source using the C-t observed data at M3 after deducting the effect of third 630 source. The error indices for both approximate and exact recovery of the third source intensity 631 function are given in Table 5. As can be seen from Figure 25 and Figure 26 and the error indices 632 of Table 5, the accuracy of the results obtained using the c-t data is slightly better than the accuracy of the results obtained using the spatial distribution of concentration data. In addition,

634 the $95 \%$ confidence interval width is narrower for the case of exact recovery, which indicates 635 less uncertainty in obtained results in this case. The main reason for this is the difference in the 636 number of observational data in these two cases. Since the spatial distribution of concentration 637 data is usually sparse and the number of available data is much less than the number of desired 638 instant for recovery of intensity function, the degree of uncertainty in retrieved results 639 increases.



640 Figure 25- Recovered intensity function of $S_{3}$ by considering the exact location of the source and 641 using observed concentration-time data at the first main station at downstream (M2) 


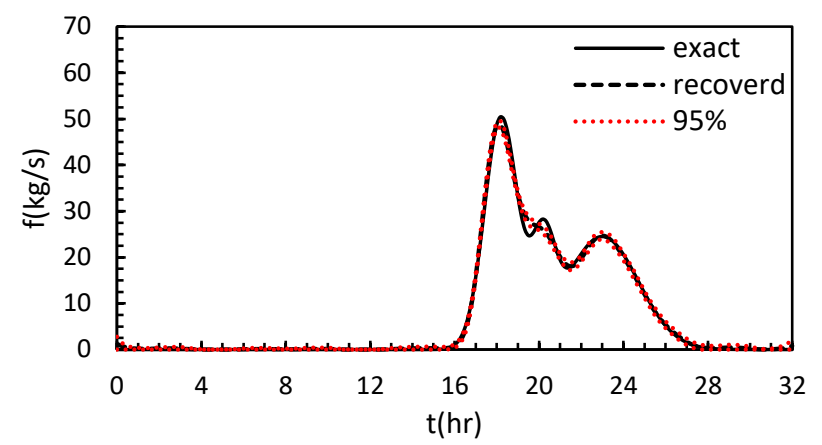

642 Figure 26- Recovered intensity function of $S_{2}$ by considering the exact location of the source and 643 using observed concentration-time data at the first main station at downstream (M3) and after revising the forward model

Table 5- Error indices of scenario 3- test 1

\begin{tabular}{|c|c|c|c|c|}
\hline \multirow[b]{2}{*}{ Index } & \multicolumn{2}{|c|}{$\mathrm{S}_{2}(46 \mathrm{~B} 1)$} & \multicolumn{2}{|c|}{$S_{3} \quad(10 B 1)$} \\
\hline & $\begin{array}{l}\text { Recovery using } \\
\text { spatial distribution of } \\
\text { concentration data }\end{array}$ & $\begin{array}{l}\text { Recovery using observed } \\
\text { concentration-time data at } \\
\text { the first main station at } \\
\text { downstream (M3) }\end{array}$ & $\begin{array}{l}\text { Recovery using } \\
\text { spatial distribution of } \\
\text { concentration data }\end{array}$ & $\begin{array}{c}\text { Recovery using } \\
\text { observed concentration- } \\
\text { time data at the first } \\
\text { main station at } \\
\text { downstream (M2) }\end{array}$ \\
\hline $\mathrm{R}^{2}(\%)$ & 95.99 & 99.64 & 98.55 & 99.96 \\
\hline $\begin{array}{l}\mathrm{RMSE} \\
(\mathrm{kg} / \mathrm{s})\end{array}$ & 2.4674 & 0.7477 & 1.5034 & 0.3585 \\
\hline $\begin{array}{c}\text { MAE }(\mathrm{kg} / \mathrm{s}) \\
\text { de }(\mathrm{kg} / \mathrm{s})\end{array}$ & $\begin{array}{c}1.4293 \\
184.7864 \\
\end{array}$ & $\begin{array}{c}0.3975 \\
14.9453 \\
\end{array}$ & $\begin{array}{c}0.9168 \\
92.1065 \\
\end{array}$ & $\begin{array}{c}0.2196 \\
24.5408 \\
\end{array}$ \\
\hline
\end{tabular}

\section{6 b) Test 2}

647 In the second case, it is assumed that the source at $10 \mathrm{~km}$ of the upstream end of the B1 starts

648 its activity earlier than the source at $46 \mathrm{~km}$ o the upstream end of the B1(Figure 27), which

649 creates a different condition in identification process than the first test. After identification of

650 the first source, similar to what described in the scenario 1, the forward model is modified

651 according to the recovered source characteristics. After revising the forward model, a

652 comparison of observed and simulated data at the main stations shows a difference between

653 these two set of data at the M2 located at $24 \mathrm{~km}$ of the upstream end of the B1 (Figure 28). 

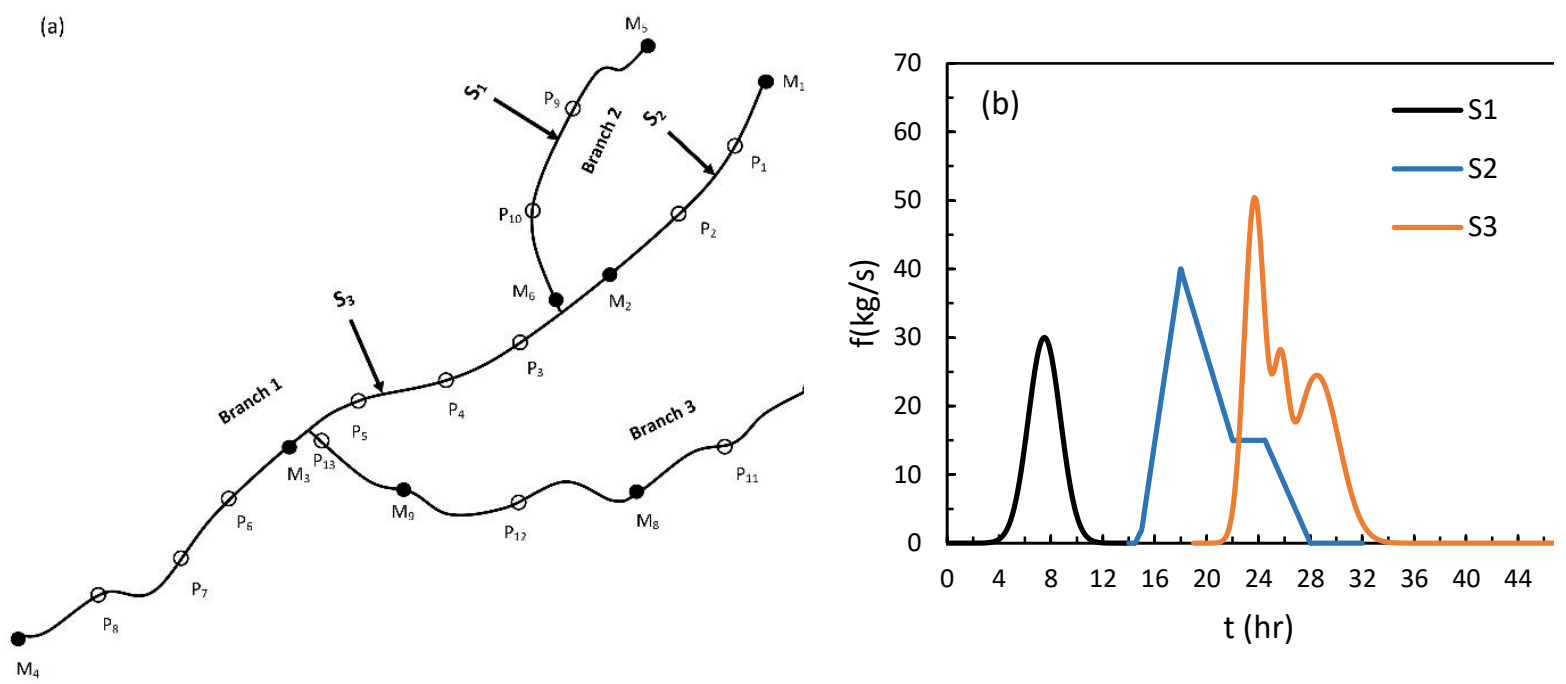

Figure 27- (a) location and (b) intensity function of pollutant sources (scenario 3-test 2)
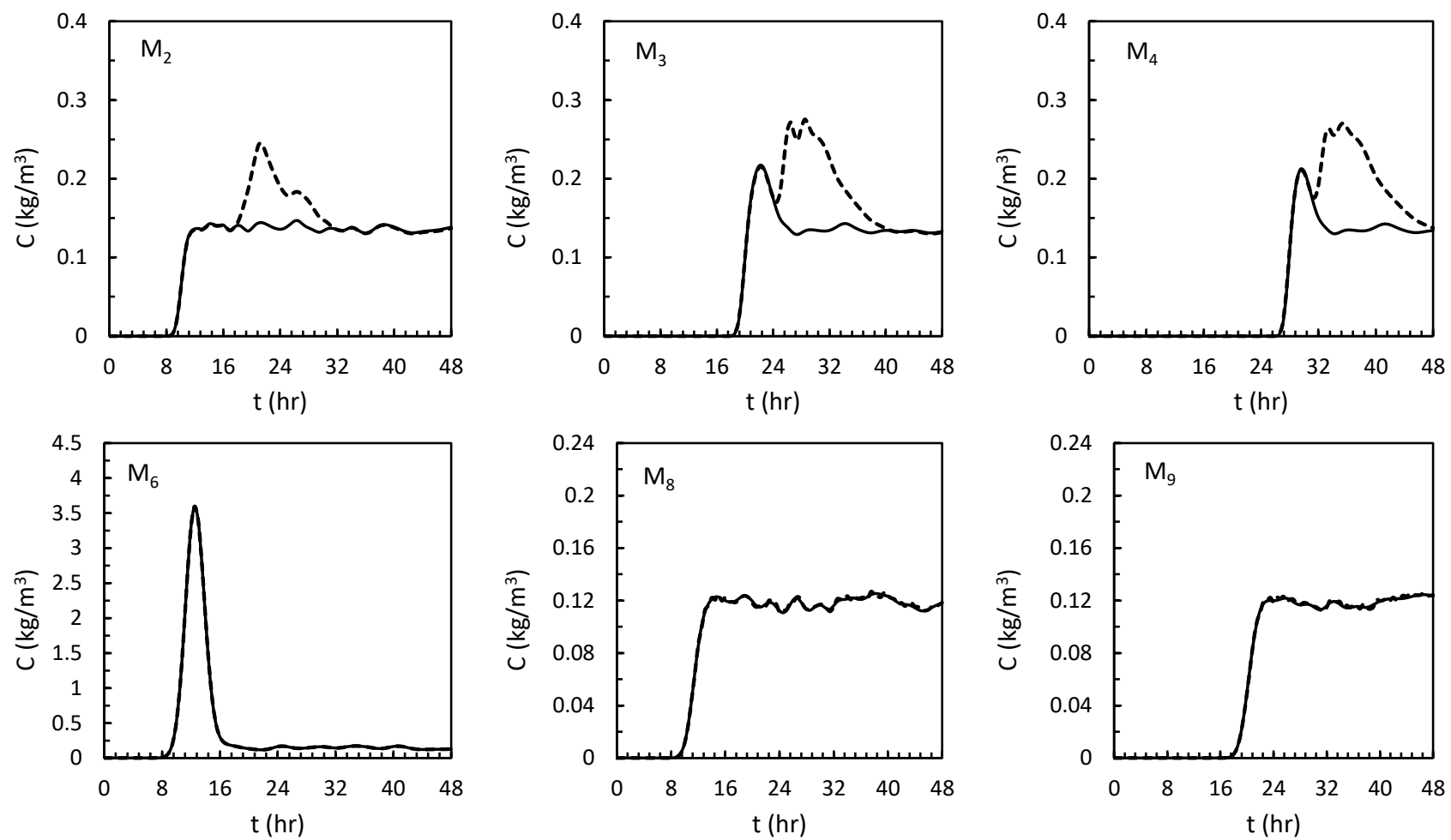

655

656

657

658

Figure 28- Comparison of simulated and observed data in main stations (scenario 3-test2) (solid line: simulated and dashed line: observed data)

Once the difference between observed and simulated data sets was detected, it is necessary to collect a concentration data at the instant of difference detection at all secondary stations located between station M2 and the first main station at upstream (namely P1 and P2), and compare those data with corresponding simulated data (Figure 29). According the Figure 29 It can be deduced that the suspected reach to presence the second source is in between P1 and P2 (i.e. at 8 to $16 \mathrm{~km}$ of the upstream end of the B1). Also, in order to ensure that there are no 
663 other simultaneously active sources upstream and downstream of the suspected reach, the 664 observed and simulated data are compared at P1 and P2 during the permanent data recording 665 period by these stations (Figure 30). As shown in Figure 30, there is no difference between the 666 observed and simulated data at P1, which means that there is no active source upstream of 667 suspected reach during the detection period. However, a comparison of these two sets of data 668 in P2 shows a difference. Regarding the general form and peak concentration of observed C-t curve with the corresponding one at M2 (Figure 28), it can be inferred that this difference is due to the discovered pollutant source and there is no other active pollutant source.

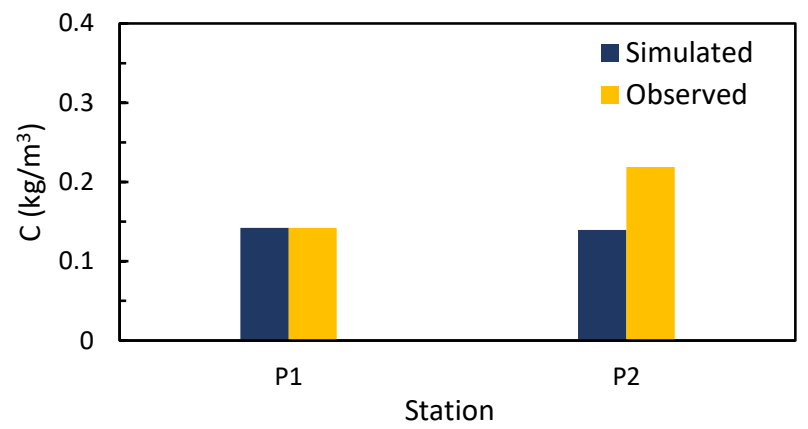

Figure 29- Comparison of simulated and observed data in secondary stations at the moment of difference detection between the simulated and observed data in M2 (scenario 3-test2)
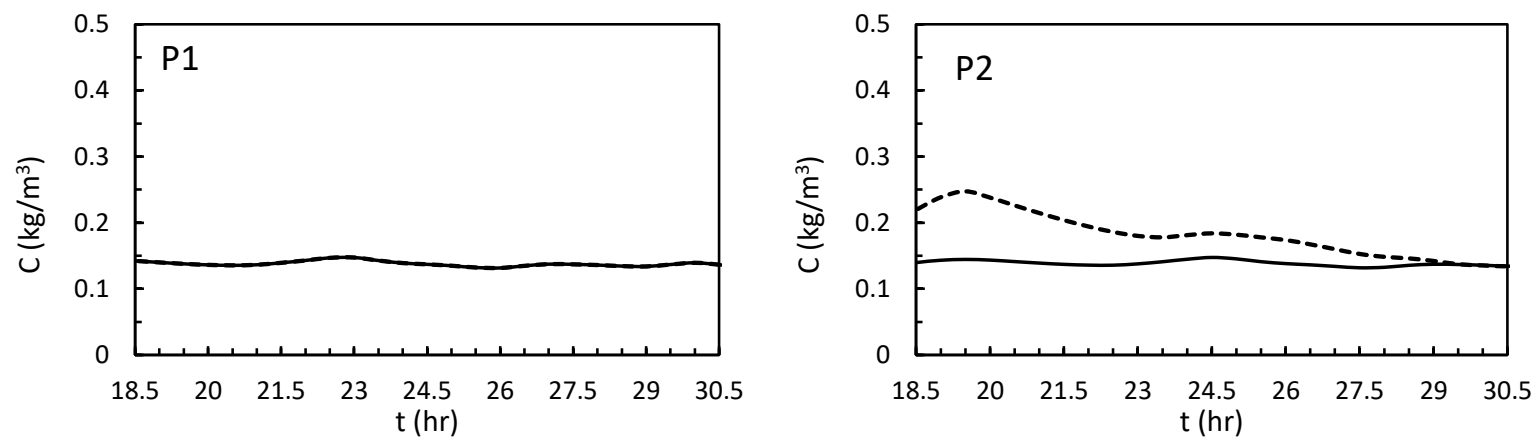

Figure 30- Comparison of observed and simulated data in secondary stations located at the upstream and downstream of suspected reach during the period of permanent data collection by those stations (scenario 3-test 2) (solid line: simulated and dashed line: observed data)

In the next step, the determined suspected reach is divided into two equal length sub-reaches of and the potential locations of the pollutant source is considered in the center of each of these

678 sub-reaches, i.e. 10 and $14 \mathrm{~km}$ of the upstream end of the B1. Then, the inverse model is implemented using spatial distribution of concentration data at all station located at 680 downstream of the suspected reach. The results of the inverse model implementation for both 
681 potential locations are presented in Figure 31 .As indicated in figure, for a potential location of

$68214 \mathrm{~km}$ the intensity function is obtained close to zero, while for a potential location of $10 \mathrm{~km} \mathrm{a}$

683 non-zero intensity is obtained. Therefore, it can be concluded that the second pollutant source

684 is located at $10 \mathrm{~km}$ from upstream of the B1, which corresponds to the assumed location.
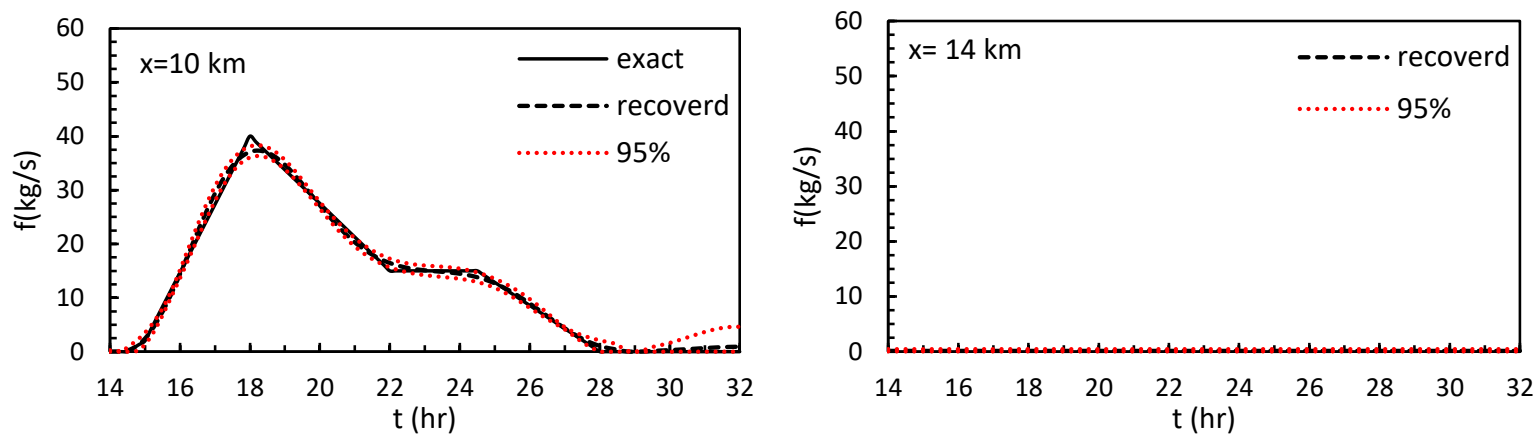

685

686

687

688

689

690

691

692

693

694

695

696

Figure 31- Recovered intensity function of $S_{2}$ at two potential locations using observed data at all main and secondary stations that located downstream of the suspected reach at the instant that the pollution cloud completely passes the $\mathrm{P} 2$ (scenario 3-test2)

After determining the exact location of the source, its intensity function are recovered more accurately, assuming the source location is known and using observed C-t data at M2 (Figure 32). The error indices for both approximate and exact recovery of the intensity function for second source are given in Table 6. As shown in Figure 32 and the error indices in Table 6, the accuracy of the results obtained using the C-t data is slightly better than the accuracy of the results obtained using the concentration spatial series data and the $95 \%$ confidence interval width is narrower as well. So, uncertainty associated with retrieved results are less in this case. The main reason for this is availability of more observation data compare to the case of recovery with spatial distribution of concentration data.

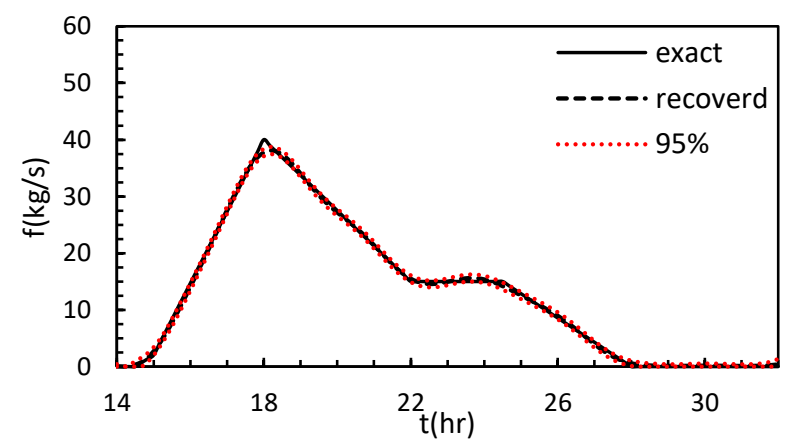
using observed concentration-time data at the first main station at downstream (M2) 
700 modified according to determined characteristics and the observed and simulated data that

701 obtained by modified forward model are compared. Comparison of these two sets of data

702 indicates the existence of difference at the M3 (Figure 33). Therefore, it can be concluded that

703 a pollution source is active upstream of this station. By comparing the concentration data at the

704 instant of difference detection in all secondary stations that located between the M3 and the

705 first main station at upstream (M2) (Figure 34), the suspected reach to presence the third

706 pollutant source is determined between 40 to $48 \mathrm{~km}$ of the upstream end of the B1.
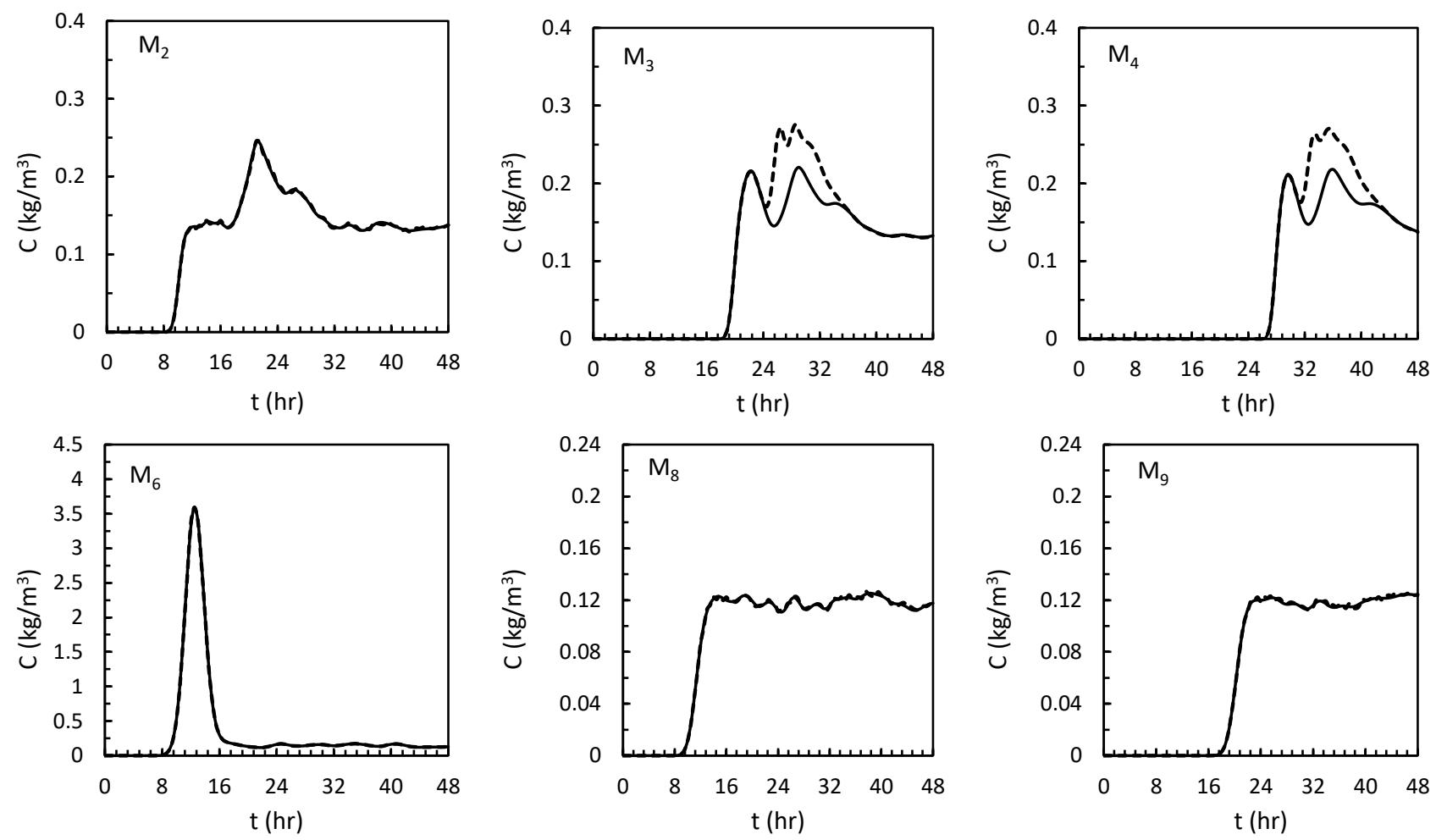

Figure 33- Comparison of simulated and observed data in main stations (scenario 3-test2) after identification of $S_{2}$ and revising the forward model (solid line: simulated and dashed line: observed 


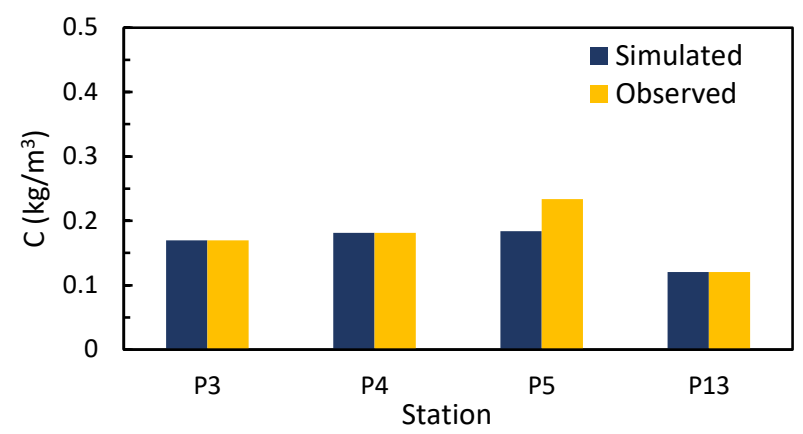

712 Figure 34- Comparison of simulated and observed data in secondary stations after identification of $\mathrm{S}_{2}$ 713 and at the instant of difference detection between the simulated and observed data in M3 (scenario 3test2)

In order to ensure that there are no other simultaneously active sources at upstream and

716 downstream of the suspected reach, the observed and simulated data at P4 and P5 secondary

717 stations are compared during the permanent data recording period by these stations(Figure 35).

718 As can be seen from Figure 35 there is no difference between the observed and simulated data

719 at $\mathrm{P} 4$, which means that there are no other active sources during the identification period. A

720 comparison of these two sets of data in the P5 shows the difference. By comparing the general

721 form and peak concentrations of Observed C-t curve with the associated one at M3 (Figure 33),

722 it can be argued that this difference is due to the discovered contaminant source and there are

723 no other active sources.
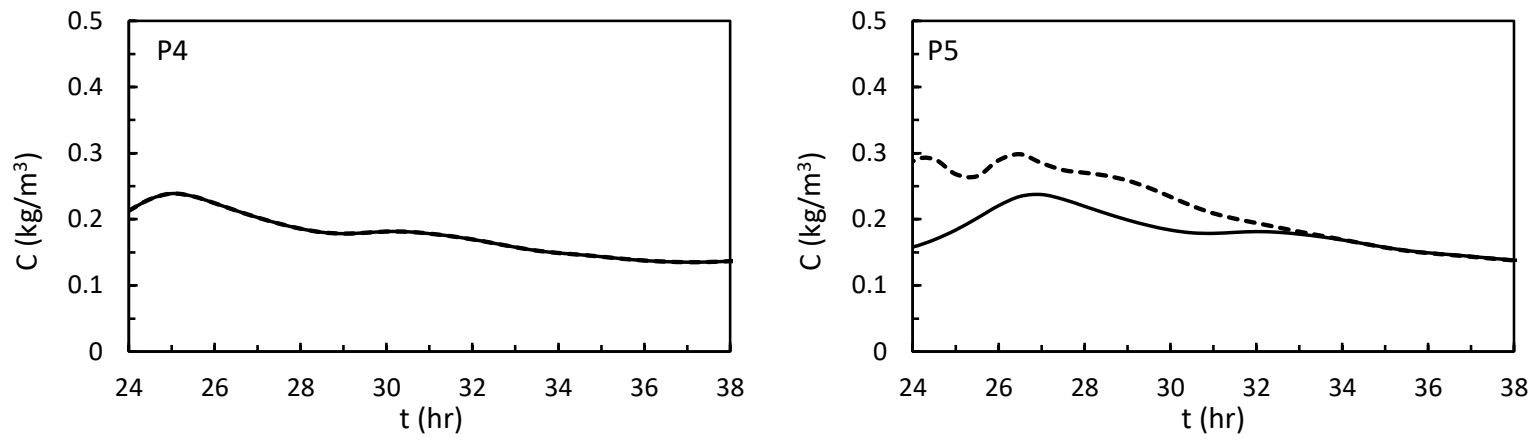

Figure 35- Comparison of observed and simulated data in secondary stations located at the upstream and downstream of suspected reach to presence of $S_{3}$ during the period of permanent data collection by those stations (scenario 3-test 2) (solid line: simulated and dashed line: observed data)

In the next step, the exact location and approximate intensity function of the third source are recovered by implementation inverse model for two potential source locations which are in the center of two equal length sub-reaches, i.e. 42 and $46 \mathrm{~km}$ of the upstream end of the B1.

730 The results have been presented in Figure 36 that shows that the location of the third source 
must be at $46 \mathrm{~km}$ of the upstream end of the B1, for which a non-zero intensity function had been obtained. However, as shown in this figure, there is no good match between the recovered and the exact intensity function. The reason for this is the time delay in identifying the effect of the third pollutant source at M3, due to the synchronization of its activity with the second pollutant source. As a result, some part of information about the third source of the pollutant is lost and consequently retrieval accuracy had been reduced and associated uncertainty increased.
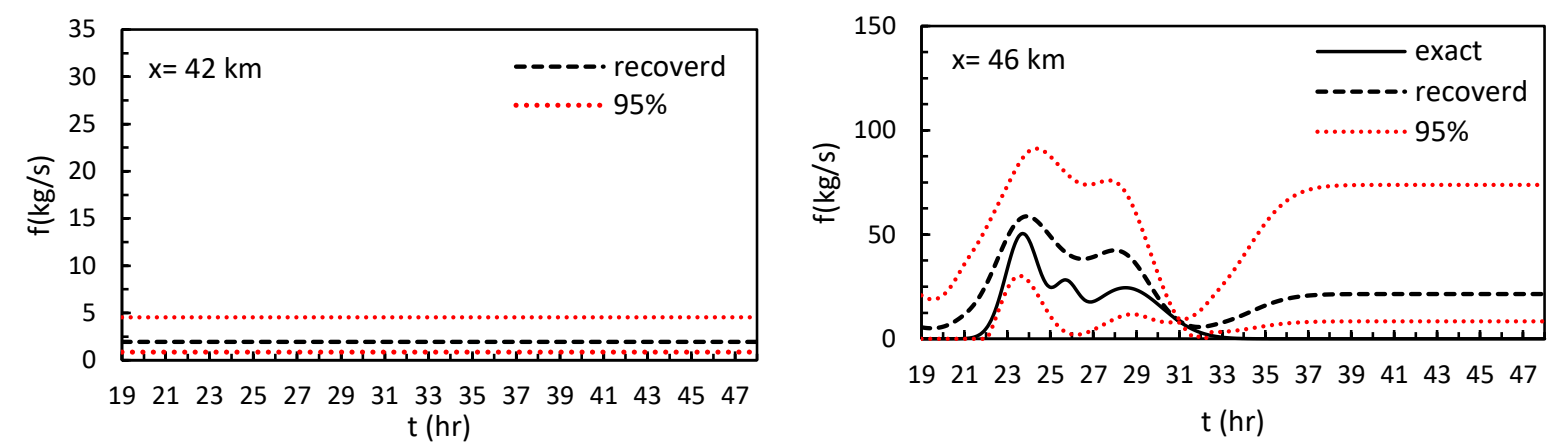

Figure 36- Recovered intensity function of $S_{3}$ at two potential locations using observed data at all main and secondary stations that located downstream of the suspected reach at the instant of recording the difference between the simulated and observed data in M3 (scenario 3-test2)

After determining the exact location of the third pollutant source, its intensity function is retrieved more accurately using the C-t data at M3 (Figure 37). As it is clear from the figure, the model has succeeded in recovering the intensity function of the mentioned pollutant source with good and acceptable accuracy. The error indices presented in Table 6also confirm this.

Figure 37- Recovered intensity function of $S_{3}$ by considering the exact location of the source and using observed concentration-time data at the first main station at downstream (M3) and after revising the forward model 
Table 6- Error indices of scenario 3- test 2

\begin{tabular}{|c|c|c|c|c|}
\hline \multicolumn{3}{|c|}{$\mathrm{S}_{2}(10 \mathrm{~B} 1)$} & \multicolumn{2}{|c|}{$S_{3} \quad(46 B 1)$} \\
\hline Index & $\begin{array}{l}\text { Recovery using } \\
\text { spatial distribution of } \\
\text { concentration data }\end{array}$ & $\begin{array}{c}\text { Recovery using } \\
\text { observed concentration- } \\
\text { time data at the first } \\
\text { main station at } \\
\text { downstream (M2) }\end{array}$ & $\begin{array}{l}\text { Recovery using } \\
\text { spatial distribution of } \\
\text { concentration data }\end{array}$ & $\begin{array}{c}\text { Recovery using } \\
\text { observed }\end{array}$ \\
\hline $\mathrm{R}^{2}(\%)$ & 99.61 & 99.88 & 74.0750 & 98.92 \\
\hline RMSE (kg/s) & 0.7702 & 0.4162 & 17.4011 & 1.3823 \\
\hline $\operatorname{MAE}(\mathrm{kg} / \mathrm{s})$ & 0.5797 & 0.3057 & 15.9637 & 0.7126 \\
\hline de $(\mathrm{kg} / \mathrm{s})$ & 20.8116 & 11.716 & 741.1620 & 52.0625 \\
\hline
\end{tabular}

753 This study has been presented an innovative multistep method for simultaneous identification

754 of the number, location and release history of pollutant source in a river network considering unsteady and non-uniform flow. The only priori information that the method needs are the expected activity period for recovery, accuracy of spatial range for retrieval the source location and the travel time of each branch. Based on those priori information, at first an adaptive arrangement of observation points is proposed. Then suspect reaches to presence of pollutant sources are delineate by comparing the simulated and observed breakthrough curves at considered stations. In this step, the number of all simultaneous active pollution sources is also determined. Then, the suspected reaches are divided to some sub-reaches and it is assumed that

762 the origin of possible sources is in the center of those sub- reaches. At the second step the

763 location and approximate release history of pollution sources are recovered by means of a 764 geostatistical approach, that considered simultaneously all the possible candidates. The source location is considered as the location where the highest amount of released pollutant is estimated. Finally, the exact release history is determined using the temporal distribution of observed concentration data at the first downstream main station.

The proposed method is suitable for practical applications, since it is based on onedimensional flow and transport models and considers the complicated real-world conditions. 
ones. Moreover, since in each simulation it is possible to identify all active pollutant sources, the required computational time is significantly lower than common iterative methods such as simulation-optimization approach. Another significant advantage of the proposed method is that it provides unique results for sought characteristics, using minimum observational data. In fact, if the observation points placed based on suggested pattern, obtaining the unique results is guaranteed. The results of application of method to a hypothetical river network for different scenarios in terms of the number, release time and location of pollutant sources, showed that the methodology performs very well in case of large-scale river networks. The given results were acceptable regarding to a limited requirement inputs. Of course, the quality of the recovery is dependent on the accuracy of the observation data. So, the uncertainty associated with results due to using erroneous observational data, was considered also through 95 percent confidence interval. This paper is one of the first attempts to solve the complicated and illposed problem of simultaneous identification of all characteristics of multiple pollutant sources in a complex river network. There are several aspects that need further investigation. Currently, the application of proposed method is limited to cases in which the activity time of pollutant sources are equal to or greater than expected activity time for recovery. Some measures such as considering random data collecting in secondary station might alleviate this problem. This is a subject for our future study.

\section{References}

Andrle, M. and El Badia, A. 2012. Identification of multiple moving pollution sources in surface waters or atmospheric media with boundary observations. Inverse problems, 28, 075009.

Atmadja, J. and Bagtzoglou, A. C. 2001. State of the art report on mathematical methods for groundwater pollution source identification. Environmental forensics, 2, 205-214.

Boano, F., Revelli, R. and Ridolfi, L. 2005. Source identification in river pollution problems: A geostatistical approach. Water resources research, 41.

Box, G. E. and Cox, D. R. 1964. An analysis of transformations. Journal of the Royal Statistical Society: Series B (Methodological), 26, 211-243. 
Butera, I., Tanda, M. G. and Zanini, A. 2013. Simultaneous identification of the pollutant release history and the source location in groundwater by means of a geostatistical approach. Stochastic Environmental Research and Risk Assessment, 27, 1269-1280.

Chapra, S. C. (2008). Surface water-quality modeling. Illinois: Waveland press.

Cheng, W. P. and Jia, Y. 2010. Identification of contaminant point source in surface waters based on backward location probability density function method. Advances in Water Resources, 33, 397-410.

De Marsily, G. 1986. Quantitative Hydrogeology: Groundwater Hydrology for Engineers Academic Press. Inc., Orlando, Florida.

El Badia, A. and Hamdi, A. 2007. Inverse source problem in an advection-dispersion-reaction system: application to water pollution. Inverse Problems, 23, 2103.

Fischer, H. B., Koh, R. C., Brooks, N. H., list, E. J. and Imberger, J. 1979. Mixing in Inland and Coastal Waters. Academic Press.

Ghane, A., Mazaheri, M. and Samani, J. M. V. 2016. Location and release time identification of pollution point source in river networks based on the Backward Probability Method. Journal of environmental management, 180, 164-171.

Gzyl, G., Zanini, A., Frączek, R. and Kura, K. 2014. Contaminant source and release history identification in groundwater: a multi-step approach. Journal of contaminant hydrology, 157, 59-72.

Hadamard, J. 1923. Lectures on Cauchy's Problem in Linear Partial Differential Equations, Yale University Press.

Hamdi, A. 2009. The recovery of a time-dependent point source in a linear transport equation: application to surface water pollution. Inverse Problems, 25, 075006.

Hamdi, A. 2016. Detection-Identification of multiple unknown time-dependent point sources in a $2 \mathrm{D}$ transport equation: application to accidental pollution. Inverse Problems in Science and Engineering, 1-25.

Hoeksema, R. J. and Kitanidis, P. K. 1985. Comparison of Gaussian conditional mean and kriging estimation in the geostatistical solution of the inverse problem. Water Resources Research, 21, 825-836.

Jiang, H. 2008. Adaptive feature selection in pattern recognition and ultra-wideband radar signal analysis. California Institute of Technology.

Kitanidis, P. K. 1995. Quasi-linear geostatistical theory for inversing. Water resources research, 31, 2411-2419.

Kitanidis, P. K. 1996. On the geostatistical approach to the inverse problem. Advances in Water Resources, 19, 333-342.

Kitanidis, P. K. and Shen, K.-F. 1996. Geostatistical interpolation of chemical concentration. Advances in Water Resources, 19, 369-378.

Lee, Y. J., Park, C. and Lee, M. L. 2018. Identification of a Contaminant Source Location in a River System Using Random Forest Models. Water, 10, 391.

Liu, C. and Ball, W. P. (1999). Application of inverse methods to contaminant source identification from aquitard diffusion profiles at Dover AFB, Delaware. Water Resources Research, 35, 1975-1985.

Mazaheri, M., Mohammad Vali Samani, J. and Samani, H. M. V. 2015. Mathematical Model for Pollution Source Identification in Rivers. Environmental Forensics, 16, 310-321.

Michalak, A. M. 2002. Environmental Contamination with Multiple Potential Sources and the Common Law: Current Approaches and Emerging Opportunities. Fordham Environmental Law Journal, 14, 147-206. 
Michalak, A. M. and Kitanidis, P. K. 2002. Application of Bayesian inference methods to inverse modelling for contaminants source identification at Gloucester Landfill, Canada. DEVELOPMENTS IN WATER SCIENCE, 47, 1259-1266.

Michalak, A. M. and Kitanidis, P. K. 2003. A method for enforcing parameter nonnegativity in Bayesian inverse problems with an application to contaminant source identification. Water Resources Research, 39.

Michalak, A. M. and Kitanidis, P. K. 2004a. Application of geostatistical inverse modeling to contaminant source identification at Dover AFB, Delaware. Journal of hydraulic research, 42, 9-18.

Michalak, A. M. and Kitanidis, P. K. 2004b. Estimation of historical groundwater contaminant distribution using the adjoint state method applied to geostatistical inverse modeling. Water Resources Research, 40.

Morrison, R. D. 2000a. Critical Review of Environmental Forensic Techniques: Part I. Environmental Forensics, 1, 157-173.

Morrison, R. D. 2000b. Critical review of environmental forensic techniques: Part II. Environmental Forensics, 1, 175-195.

Neupauer, R. M., Borchers, B. and Wilson, J. L. 2000. Comparison of inverse methods for reconstructing the release history of a groundwater contamination source. Water Resources Research, 36, 2469-2475.

Skaggs, T. H., and Z. J. Kabala. (1994). Recovering the release history of a groundwater contaminant. Water Resour. Res, 30(1), 71- 79.

Skaggs, T. H. and Kabala, Z. 1998. Limitations in recovering the history of a groundwater contaminant plume. Journal of Contaminant Hydrology, 33, 347-359.

Snodgrass, M. F. and Kitanidis, P. K. 1997. A geostatistical approach to contaminant source identification. Water Resources Research, 33, 537-546.

Taylor, G. 1954. The dispersion of matter in turbulent flow through a pipe. Proceedings of the Royal Society of London. Series A. Mathematical and Physical Sciences, 223, 446-468.

Telci, I. T. and Aral, M. M. 2011. Contaminant source location identification in river networks using water quality monitoring systems for exposure analysis. Water Quality, Exposure and Health, 2, 205-218.

Wang, J., Zhao, J., Lei, X. and Wang, H. 2018. New approach for point pollution source identification in rivers based on the backward probability method. Environmental Pollution, 241, 759-774.

Woodbury, A., Sudicky, E., Ulrych, T. J. and Ludwig, R. 1998. Three-dimensional plume source reconstruction using minimum relative entropy inversion. Journal of Contaminant Hydrology, 32, 131-158.

$\mathrm{Wu}, \mathrm{W} .2007$. Computational river dynamics, CRC Press.

Yang, H., Shao, D., Liu, B., Huang, J. and Ye, X. 2016. Multi-point source identification of sudden water pollution accidents in surface waters based on differential evolution and Metropolis-Hastings-Markov Chain Monte Carlo. Stochastic environmental research and risk assessment, 30, 507-522. 
894 Declarations

895

896 Funding

897 No funding was received for conducting this study.

898 Conflicts of interest/Competing interests

899 The authors declare that they have no conflicts of interest.

$900 \quad$ Availability of data and material

$901 \quad$ Not applicable.

902 Code availability

903 Not applicable.

904 